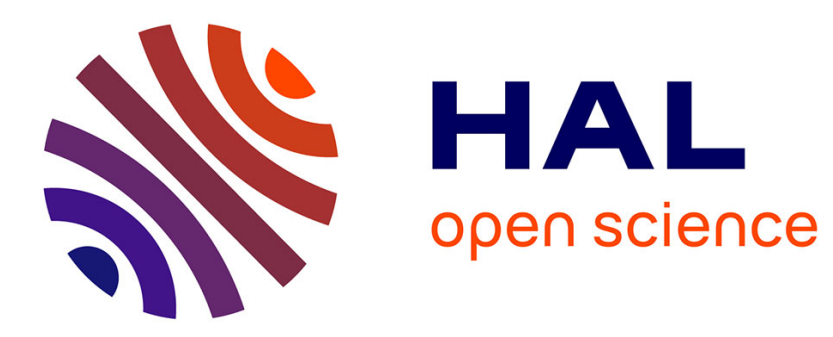

\title{
Periodic solutions of a two-degree-of-freedom autonomous vibro-impact oscillator with sticking phases
}

\author{
Huong Le Thi, Stéphane Junca, Mathias Legrand
}

\section{To cite this version:}

Huong Le Thi, Stéphane Junca, Mathias Legrand. Periodic solutions of a two-degree-of-freedom autonomous vibro-impact oscillator with sticking phases. Nonlinear Analysis: Hybrid Systems, 2018, 28, pp.54-74. 10.1016/j.nahs.2017.10.009 . hal-01305719v2

\section{HAL Id: hal-01305719 \\ https://hal.science/hal-01305719v2}

Submitted on 19 Jan 2018

HAL is a multi-disciplinary open access archive for the deposit and dissemination of scientific research documents, whether they are published or not. The documents may come from teaching and research institutions in France or abroad, or from public or private research centers.
L'archive ouverte pluridisciplinaire HAL, est destinée au dépôt et à la diffusion de documents scientifiques de niveau recherche, publiés ou non, émanant des établissements d'enseignement et de recherche français ou étrangers, des laboratoires publics ou privés.

\section{다(1)(2)}

Distributed under a Creative Commons Attribution - ShareAlikel 4.0 International 


\title{
PERIODIC SOLUTIONS OF A TWO-DEGREE-OF-FREEDOM AUTONOMOUS VIBRO-IMPACT OSCILLATOR WITH STICKING PHASES
}

Huong LE THI, Université Côte d'Azur, LJAD, CNRS \& Inria, Nice, France \& Department of Mathematics and Informatics, Thang Long University, Ha Noi City, Viet Nam.

Stéphane JUNCA, Université Côte d'Azur, LJAD, CNRS \& Inria, Nice, France.

Mathias LEGRAND, Department of Mechanical Engineering, McGill University, Montréal, Québec, Canada.

\begin{abstract}
This contribution explores the free dynamics of a simple two-degree-of-freedom vibro-impact oscillator. One degree-of-freedom is unilaterally constrained by the presence of a rigid obstacle and periodic solutions involving one sticking phase per period (1-SPP) are targeted. A solution method to obtain such orbits is proposed: it provides conditions on the existence of 1-SPP as well as closed-form solutions. It is shown that 1-SPP might not exist for a given combination of masses and stiffnesses. The set of 1-SPP is at most a countable set of isolated periodic orbits. The construction of 1-SPP requires numerical developments that are illustrated on a few relevant examples. Comparison with nonlinear modes of vibration involving one impact per period (1-IPP) is also considered. Interestingly, an equivalence between 1-SPP and a special set of isolated 1-IPP is established. It is also demonstrated that the prestressed system features sticking phases of infinite duration.
\end{abstract}

KEYWORDS. vibration; impact; sticking phase; periodic solutions.

\section{INTRODUCTION}

In the context of vibration and modal analysis of vibro-impact oscillators, nonlinear modes of vibration with non-grazing impact are explored in $[15,13,9,11,19,17]$. In these works, a single degree-of-freedom (dof) is unilaterally constrained by the presence of a rigid foundation: the dynamics is purely linear when the contact constraint is not active, and governed by an impact law otherwise. Accordingly, the contact force arising when the system interacts with the foundation is a periodic distribution of Dirac deltas.

Instead, the present work pays attention to periodic solutions involving long "sticking" contact phases, thus discarding impulse-driven dynamics reported in the previous works, during which the contacting mass rests against the obstacle for a finite amount of time. Let us clarify the terminology now: as explained later, "sticking" here means that the impacting mass will rest on the rigid foundation for a finite or infinite amount of time during its motion even though there is no "adhesion force" arising from the rigid foundation and acting on the impacting mass. An equivalent terminology would be "lasting non-impulsive" contact phases. This investigation is motivated by the fact that in a continuous setting in space and time, unilateral contact forces are known to be discontinuous functions at most [8] while they become impulsive after a semi-discretization in space via the Finite Element technique. The question is then: are there non-impulsive solutions in the Finite Element framework and alike?

The $N$-degree-of-freedom case has been recently investigated in [18] with another approach. Some important features of the singular dynamics near contacts with zero velocity have been presented in $[7,10]$.

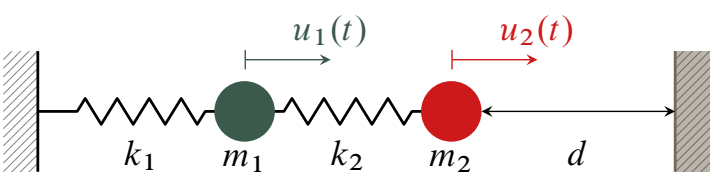

FIGURE 1. Two degree-of-freedom vibro-impact system at equilibrium, $d>0$

The system of interest is an oscillator with two masses $m_{1}$ and $m_{2}$ linearly connected through two springs of stiffness $k_{1}$ and $k_{2}$ respectively, as depicted in Figure 1. The dynamics of interest reads:

$$
\begin{aligned}
& \mathbf{M u ̈}+\mathbf{K u}=\mathbf{r} \\
& \mathbf{u}(0)=\mathbf{u}_{0}, \quad \dot{\mathbf{u}}(0)=\dot{\mathbf{u}}_{0} \\
& u_{2}(t) \leq d, \quad R(t) \leq 0, \quad\left(u_{2}(t)-d\right) R(t)=0, \quad \forall t \\
& \dot{\mathbf{u}}^{\top} \mathbf{M} \dot{\mathbf{u}}+\mathbf{u}^{\top} \mathbf{K u}=\mathbf{E}(t)=\mathbf{E}(0)
\end{aligned}
$$


where

$$
\mathbf{M}=\left[\begin{array}{cc}
m_{1} & 0 \\
0 & m_{2}
\end{array}\right], \quad \mathbf{K}=\left[\begin{array}{cc}
k_{1}+k_{2} & -k_{2} \\
-k_{2} & k_{2}
\end{array}\right], \quad \mathbf{u}(t)=\left(\begin{array}{l}
u_{1}(t) \\
u_{2}(t)
\end{array}\right) \quad \text { and } \quad \mathbf{r}(t)=\left(\begin{array}{c}
0 \\
R(t)
\end{array}\right) .
$$

Above, $u_{j}, \dot{u}_{j}$, and $\ddot{u}_{j}$ represent the displacement, velocity, and acceleration of mass $j, j=1,2$, respectively. The fixed gap $d$ is defined between the obstacle and the equilibrium position of the second mass. It is the algebraic distance between mass 2 at rest without any external force and the rigid wall and might thus be negative in the prestressed case. The quantity $R(t)$ is the reaction force of the wall on mass 2 .

Matrices $\mathbf{M}$ and $\mathbf{K}$ are symmetric positive definite so that there is a matrix $\mathbf{P}$ of eigenmodes which simultaneously diagonalizes both of them, that is $\mathbf{P}^{\top} \mathbf{M P}=\mathbf{I}$ and $\mathbf{P}^{\top} \mathbf{K P}=\boldsymbol{\Omega}^{2}=\left.\boldsymbol{d i a g}\left(\omega_{i}^{2}\right)\right|_{i=1,2}$ where I is the $2 \times 2$ identity matrix and $\omega_{i}^{2}, i=1,2$ are the eigenfrequencies of the linear system without unilateral contact.

Equation (1.1d) reflects the conservative nature of the system. Energy is preserved along a motion. Equation (1.1d) implies the existence of a perfectly elastic impact law of the form $\dot{u}_{2}^{+}=-e \dot{u}_{2}^{-}$with $e=1$ where $\dot{u}_{2}^{-}$and $\dot{u}_{2}^{+}$respectively stand for the pre- and post-impact velocities of mass 2 . As detailed later, 1-SPP orbits are defined to be independent of the restitution coefficient $e$. Thus 1-SPP still exist for $e \in[0 ; 1]$. However, the framework $e=1$ is chosen for comparison with another class of periodic solutions with nonzero velocity at the impact. Moreover, the structure of a general solution is simpler when $e=1$. This initial-value problem with conserved energy is known to be well-posed [14, 4]. The sticking phase is known to appear as a limit of a chattering sequence $[3,7,6,12]$. However in the present work, there is no source term and sticking periodic solutions can still occur.

The paper is organized as follows. In Section 2, the definition of a "sticking phase" is provided and conditions for its occurrence are stated. Then, necessary conditions satisfied by periodic solutions with one sticking phase per period (1-SPP) are given in terms of the free flight duration $s$. The parameter $s$ appears to be the key parameter and a root of an explicit nonlinear function. Furthermore, when $s$ is known, the corresponding 1-SPP is expressed in closed form. The method and numerical examples are described in Section 3 in order to find all 1-SPP.

Mathematical proofs are detailed in Section 4, 5 and 6. More precisely, Section 4 deals with the structure of the space of solutions with a sticking phase. Section 5 is devoted to prove Theorem 2.1 on 1-SPP. The existence result of an infinite set of admissible initial data such that the associated solutions satisfy the constraint $u_{2} \leq d$ near the sticking phase is proven in Section 6. The existence of 1-SPP satisfying the constraint $u_{2} \leq d$ during the whole period remains an open problem. The prestressed case with $d \leq 0$ is discussed in Section 7. Section 8 concludes the paper. In this work, all numerical simulations are performed using the parameters $m_{1}=m_{2}=1$ and $k_{1}=k_{2}=1$, unless indicated otherwise.

\section{MAIN RESULTS}

The occurrence of a sticking phase is first defined with necessary and sufficient conditions in Section 2.1. Then, in Section 2.2, periodic solutions with one sticking phase per period, the so-called 1-SPP, are characterized through necessary conditions to exhibit them all. Throughout the current work, internal resonances are discarded, i.e. $\cap_{j=1}^{2} T_{j} \mathbb{N}=\emptyset$, unless stated otherwise.

2.1. Sticking phase. A sticking phase occurs when the mass number 2 stays at $u_{2}=d$ on a proper time interval.

Definition 2.1 - Sticking phase and its duration. Let $\mathbf{u}$ be a solution to System (1.1). A sticking phase arises if there exist $t_{0} \in \mathbb{R}$ and $\mathcal{T}>0$ such that

$$
u_{2}(t)=d, \quad \forall t \in\left[t_{0} ; t_{0}+\mathcal{T}\right] .
$$

Moreover, when there exists $0<\delta \ll 1$ such that $\forall t \in] 0 ; \delta[$

$$
u_{2}\left(t_{0}-t\right)<d \text { and } u_{2}\left(t_{0}+\mathcal{T}+t\right)<d,
$$

then $t_{0}$ is the starting time and $\mathcal{T}$ is the duration of the sticking phase. 
The central reference [4] is used throughout the paper: existence is recalled, uniqueness and continuous dependence to the initial data is proved. Moreover, in the conservative case, it is shown that there is no impact accumulation. Accordingly, before and after the sticking phase, condition (2.2) above is sufficient to properly define the beginning and the end of a sticking phase of finite duration. More precisely, it is proven in Proposition 19 of [4] that the impact times are isolated for the perfectly elastic impact law. It does not hold true when $0<e<1$ since chattering might occur [7].

In the present work, the finite duration of the sticking phase for $d>0$ is a consequence of Theorem 2.1 . This is not always true, as for example with the prestressed case $d \leq 0$ detailed in Section 7. Such conditions are known to be related to the sign of the acceleration [7] just before impact and are already precisely established for our two-dof system [9].

Theorem 2.1 - Sticking contact. Assume $d>0$. There exists a sticking phase exactly starting at time $t_{0}$ and persisting on its right neighbhourhood if and only if:

(1) $u_{2}\left(t_{0}\right)=d, \dot{u}_{2}^{-}\left(t_{0}\right)=0, u_{1}\left(t_{0}\right)>d$, or

(2) $u_{2}\left(t_{0}\right)=d, \dot{u}_{2}^{-}\left(t_{0}\right)=0, u_{1}\left(t_{0}\right)=d, \dot{u}_{1}\left(t_{0}\right)>0$.

The second case where $u_{1}\left(t_{0}\right)=d$ and $\dot{u}_{1}\left(t_{0}\right)>0$ corresponds to the beginning of the sticking phase. The duration of the sticking phase $\mathcal{T}$ then only depends on

$$
\begin{aligned}
v & =\dot{u}_{1}\left(t_{0}\right), \quad \text { and } \\
\mathcal{T}(v) & =\frac{2}{\omega} \arctan (\xi v) \quad \text { where } \omega=\sqrt{\frac{k_{1}+k_{2}}{m_{1}}} \text { and } \xi=\frac{\sqrt{\left(k_{1}+k_{2}\right) m_{1}}}{k_{1} d} .
\end{aligned}
$$

The state of system at the end of the sticking phase is:

$$
u_{2}\left(t_{0}+\mathcal{T}\right)=d, \quad \dot{u}_{2}^{-}\left(t_{0}+\mathcal{T}\right)=0, \quad u_{1}\left(t_{0}+\mathcal{T}\right)=d, \quad \dot{u}_{1}\left(t_{0}+\mathcal{T}\right)=-v .
$$

Moreover, the regularity of the curve $\left\{\left(u_{2}(t), \dot{u}_{2}(t)\right), t \in \mathbb{R}\right\}$ is $C^{1.5}$ at the point $\left(u_{2}, \dot{u}_{2}\right)=(d, 0)$ which corresponds to the time interval $\left[t_{0}, t_{0}+\mathcal{T}\right]$.

The starting of the sticking phase corresponding to the second case stated in Theorem 2.1 is only true for $e=1$ since there is the uniqueness of solution in the past for $e=1$ [4]. For $e<1$, the beginning of the sticking phase can be for $u_{1}\left(t_{0}\right)>d$. It is easy to see for $e=0$. The proof of this theorem is written in Section 4. The space $C^{s}$ is the Sobolev space $W^{s, \infty}$ when $s$ is a fractional number [2] which is a generalization of Hölder spaces for $s>1$. It should also be highlighted that there is no unilateral constraint on mass 1 . The expression $u_{1}>d$ sometimes used below does not mean that the mass 1 hits wall since $u_{1}$ is the displacement and not the position of mass 1 . In the sequel, we do not restrain $u_{1}$.

The loss of regularity at the sticking point is quite clear in Figure 2. This is the least smooth point on the curve $\left\{\left(u_{2}(t), \dot{u}_{2}(t)\right), t \in \mathbb{R}\right\}$. Locally, the orbit is very similar to the graph of $t \mapsto\left(d-|t|^{1.5}, t\right)$. Elsewhere, the curve is analytic.

Incidentally, the proof of Theorem 2.1 leads to an explicit classification of all possible contact patterns. There is no accumulation of impacts and only three distinct contact configurations arise. At $t=t_{0}$, let us assume $u_{2}\left(t_{0}\right)=d$, then contact is

(1) an impact if $\dot{u}_{2}^{-}\left(t_{0}\right)>0$;

(2) a grazing contact if $\dot{u}_{2}\left(t_{0}\right)=0$ and $R(t)=0$ for all $t \approx t_{0}$;

(3) a sticking contact if $\dot{u}_{2}\left(t_{0}\right)=0$ and $R(t)<0$ for some $t \approx t_{0}$.

Since the velocity of mass 2 shall be discontinuous, $\dot{u}_{2}^{ \pm}\left(t_{0}\right)$ denotes its left/right limit. Due to energy conservation, $\dot{u}_{2}^{+}\left(t_{0}\right)=-\dot{u}_{2}^{-}\left(t_{0}\right)$ in our model; in particular, when the incoming velocity vanishes, that is $\dot{u}_{2}^{-}\left(t_{0}\right)=\dot{u}_{2}^{+}\left(t_{0}\right)=\dot{u}_{2}\left(t_{0}\right)=0$, the velocity is continuous.

Sticking contact and grazing contact are the two possible contact occurrences [7] for an impact with zero velocity. In general, it is challenging to know whether a zero velocity impact generates a reaction of the wall without further information. For the considered two-dof system, simple conditions are given on the position and velocity of mass 1 to distinguish a grazing contact from a sticking contact.

(1) An impact yields an instantaneous rebound with $\dot{u}_{2}^{+}\left(t_{0}\right)=-\dot{u}_{2}^{-}\left(t_{0}\right)<0$.

(2) A grazing contact means that the trajectory would not change irrespective of the presence of the wall. As a corollary of Theorem 2.1, the data at time $t_{0}$ is either $u_{1}\left(t_{0}\right)<d, u_{2}\left(t_{0}\right)=d$, and $\dot{u}_{2}\left(t_{0}\right)=0$ or $u_{1}\left(t_{0}\right)=d, \dot{u}_{1}\left(t_{0}\right) \leq 0, u_{2}\left(t_{0}\right)=d$, and $\dot{u}_{2}\left(t_{0}\right)=0$. Moreover, there exists $\varepsilon>0$ such that $R(t)=0$ for all $t \in] t_{0}-\varepsilon ; t_{0}+\varepsilon[$ 

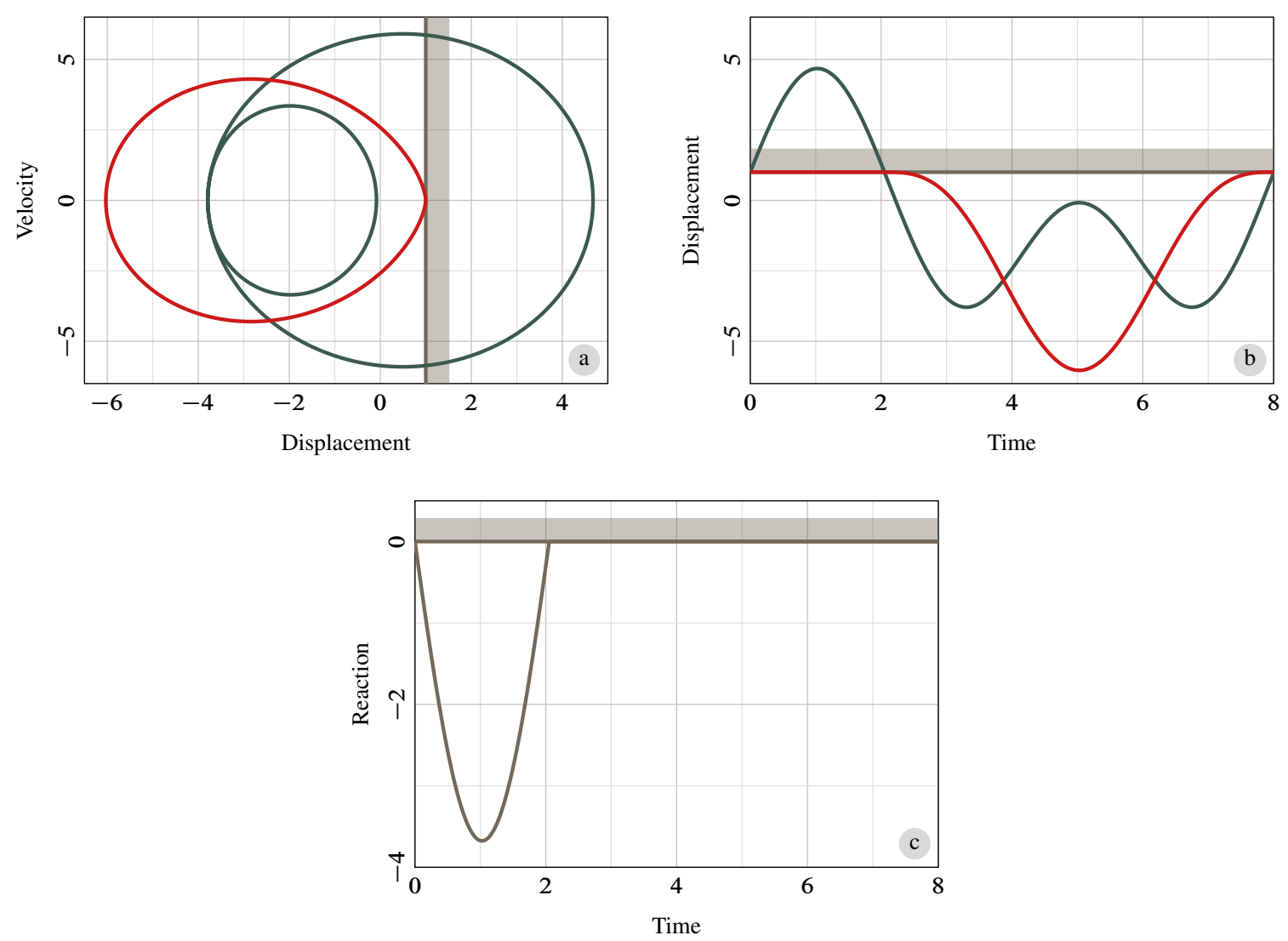

FIGURE 2. Admissible 1-SPP with a singularity 1.5 at the intersection between the red trajectory and the wall, $d=1$ and initial data $U(0)=[1,1,5.8624394,0]$. (a) Orbits $\left\{\left(u_{i}(t), \dot{u}_{i}(t)\right), t \in \mathbb{R}\right\}, i=1$, 2. (b) Displacements $t \mapsto u_{i}(t), i=1$, 2. (c) Reaction of the wall $t \mapsto R(t)$

(3) A sticking contact can be divided into three sequential steps:

(a) the beginning of a sticking contact: $u_{1}\left(t_{0}\right)=d, \dot{u}_{1}\left(t_{0}\right)>0, u_{2}\left(t_{0}\right)=d$, and $\dot{u}_{2}\left(t_{0}\right)=0$. There exists $\varepsilon>0$ such that $R(t)=0$ for all $t \in] t_{0}-\varepsilon ; t_{0}[$ and $R(t)<0$ for all $t \in] t_{0} ; t_{0}+\varepsilon$ [;

(b) the resting phase of a sticking contact: $u_{1}\left(t_{0}\right)>d, u_{2}\left(t_{0}\right)=d$, and $\dot{u}_{2}\left(t_{0}\right)=0$. There exists $\varepsilon>0$ such that $R(t)<0$ for all $t \in] t_{0}-\varepsilon ; t_{0}+\varepsilon[$;

(c) the end of a sticking contact: $u_{1}\left(t_{0}\right)=d, \dot{u}_{1}\left(t_{0}\right)<0, u_{2}\left(t_{0}\right)=d$, and $\dot{u}_{2}\left(t_{0}\right)=0$. There exists $\varepsilon>0$ such that $R(t)<0$ for all $t \in] t_{0}-\varepsilon ; t_{0}[$ and $R(t)=0$ for all $t \in] t_{0} ; t_{0}+\varepsilon[$.

2.2. Periodic solutions with one sticking phase per period. The main result of this paper is concerned with the possible existence and computation of 1-SPP.

Definition 2.2 - One sticking phase per period solution. A function $\mathbf{u}$ is called a 1-SPP, if there exist $0<\mathcal{T}<T$ such that $\mathbf{u}$ is a $T$-periodic solution to (1.1) with one sticking phase per period, that is to say up to a time translation that

(1) $u_{2}=d$ on $[0 ; \mathcal{T}]$,

(2) $u_{2}<d$ on $] \mathcal{T} ; T[$, and

(3) $\mathbf{u}(T)=\mathbf{u}(0)$ and $\dot{\mathbf{u}}^{-}(T)=\dot{\mathbf{u}}^{-}(0)$.

Condition (2) above can be relaxed to $u_{2}(t) \leq d$ on $] \mathcal{T} ; T$ [ only. This yields admissible periodic solutions with potentially many grazing contacts and sticking phases.

In order to find and characterize all 1-SPP, the following notations are needed:

$$
\mathbf{P}=\left[\begin{array}{ll}
P_{11} & P_{12} \\
P_{21} & P_{22}
\end{array}\right], \quad \mathbf{B}=\mathbf{P}^{-1}=\left[\begin{array}{ll}
B_{11} & B_{12} \\
B_{21} & B_{22}
\end{array}\right]
$$




$$
\begin{aligned}
& \Phi_{j}(s)=\frac{\sin \left(\omega_{j} s\right)}{\omega_{j}\left(1-\cos \left(\omega_{j} s\right)\right)}=\frac{1}{\omega_{j}} \cot \left(\frac{\omega_{j} s}{2}\right), \\
& a_{k j}=-P_{k j} B_{j 1}, \quad b_{k j}=\frac{a_{k j}}{\omega_{j}}, \quad \alpha_{j}=b_{1 j}-b_{2 j}, \quad \beta_{j}=b_{1 j}, \\
& w_{k}(s)=\sum_{j=1}^{2} a_{k j} \Phi_{j}(s)=\sum_{j=1}^{2} b_{k j} \cot \left(\frac{\omega_{j} s}{2}\right),
\end{aligned}
$$

with $j=1,2$ and $k=1,2$. Note that the interaction coefficients $a_{k j}$ in [11] and in this work have opposite sign. If a 1-SPP exists, then there is only one control parameter, the duration of the free flight $s$, which uniquely determines the 1-SPP through Theorem 2.2. The initial data and the period $T$ are functions of $s$. Conversely, such initial data may generate a ghost solution [15] if $u_{2}$ becomes greater than $d$ during the free flight.

The natural way to obtain periodic solutions for System (1.1) is to look for the fixed points of the associated first return map (FRM). From an quasi-explicit expression of the FRM, it appears that closed forms except for an unknown parameter: the free flight time which is a root of an explicit function $h$. The roots of $h$ are carefully explored to obtain a countably infinite set of initial data which yields 1-SPP if and only if the constraint $u_{2}<d$ is satisfied during the free flight. This approach generalizes to the prestressed case $d \leq 0$.

Theorem 2.2 - 1-SPP characterization. Assume $\mathbf{u}$ is a 1-SPP of System (1.1), then:

(1) The duration of the free flight $s>0$ is necessarily a root of

$$
h(s)=w_{1}(s)-w_{2}(s)=\sum_{j=1}^{2} \alpha_{j} \cot \left(\frac{\omega_{j} s}{2}\right)=0 .
$$

(2) The solution $\mathbf{u}$ corresponds to the initial data

$$
\left[u_{1}(0), u_{2}(0), \dot{u}_{1}(0), \dot{u}_{2}(0)\right]=[d, d, v, 0] \text { where } v=v(s)=d / w_{1}(s) .
$$

(3) The period $T$ of $\mathbf{u}$ is a function of $s: T(s)=s+\mathcal{T}(v(s))$ where $\mathcal{T}$ is defined in (2.3).

(4) The orbit is symmetric: $\mathbf{u}(\theta+t)=\mathbf{u}(\theta-t), \forall t$ where $\theta:=\mathcal{T}+s / 2$.

The proof of this theorem is given in Sections 5.1 and 5.2.

Remark 2.1. Instead of $s$, the parameter characterizing a 1-SPP could be the velocity $v$ of the first mass at the beginning of the sticking phase. Choosing $v$ specifies all the initial data (2.10) at the beginning of the sticking phase for a 1-SPP and then the 1-SPP for all time. Accordingly, there is a one-to-one correspondence between the set of 1-SPP and the set of initial velocity $v=\dot{u}_{1}\left(t_{0}\right)$ which yields a 1-SPP. However, the 1-SPP closed-form expressions are simpler with $s$ which is chosen in the remainder.

Remark 2.2. The set of 1-SPP is at most countable and corresponds to a subset of roots of the analytic function $h(\cdot)$ defined in Equation (2.9).

The roots of the quasi-periodic function $h(\cdot)$ are the first ingredients to be investigated to look for 1-SPP. In addition, the velocity of the first mass at the beginning of the sticking phase has to be positive, see Theorem 2.1. The sign of this velocity is governed by the sign of $w_{1}(s)$.

The sticking phase can now be exactly computed. Without loss of generality, assume $t_{0}=0$. The end of the sticking phase is the beginning of the free flight. Denoting $\underline{\mathbf{u}}$ the free flight portion of the solution with $\mathcal{T}=\mathcal{T}(s)$ leads to

$$
\begin{aligned}
& (\underline{\mathbf{u}}, \underline{\mathbf{\mathbf { u }}})(\mathcal{T}):=(\mathbf{u}, \dot{\mathbf{u}})(\mathcal{T}) \\
& \mathbf{M} \underline{\mathbf{u}}(t)+\mathbf{K} \underline{\mathbf{u}}(t)=0, \quad \forall t \in] \mathcal{T} ; T[.
\end{aligned}
$$

A solution to Equations (2.11)-(2.12) is a physically admissible solution to System (1.1) if it satisfies the constraint

$$
\underline{u}_{2}(t)<d, \quad \mathcal{T}<t<T .
$$


If condition (2.13) is violated, then the 1-SPP is not admissible: this is a "ghost" solution [15]. Hence, introducing the following sets:

$$
\begin{aligned}
Z & =\{s>0, h(s)=0\}, \\
Z^{-} & =\left\{s \in Z \text { and } w_{1}(s)<0\right\}, \\
Z^{0} & =\left\{s \in Z \text { and } w_{1}(s)=0\right\}, \\
Z^{+} & =\left\{s \in Z \text { and } w_{1}(s)>0\right\}, \\
Z^{\text {ad }} & =\left\{s \in Z^{+} \text {and }(2.13) \text { is satisfied }\right\} \subset Z^{+}
\end{aligned}
$$

and $Z=Z^{+} \cup Z^{0} \cup Z^{-}$, the admissible free flight times $s$ belongs to $Z^{+}$which also corresponds to the "admissible" initial data. Furthermore, from the admissible initial data, the set of admissible 1-SPP has a one-to-one correspondence with $Z^{\text {ad }}$. Is $Z^{\text {ad }}$ empty or not? It is not easy to answer due to the global constraint (2.13) during the full free flight. However, we can quantify the size of $Z^{+}$which leads to solutions satisfying (2.13) at least near the sticking phase. The following assumption 2.1 is needed to avoid that $Z^{+}=\emptyset$, see Section 6 below.

Assumption 2.1. $\operatorname{det}\left(\begin{array}{ll}\alpha_{1} & \alpha_{2} \\ \beta_{1} & \beta_{2}\end{array}\right) \neq 0$.

Notice that if Assumption 2.1 is violated and $\left(\alpha_{1}, \alpha_{2}\right) \neq(0,0),\left(\beta_{1}, \beta_{2}\right) \neq(0,0)$ then $h(\cdot)$ and $w_{1}(\cdot)$ have the same roots, i.e. $Z=Z^{0}$, and $Z^{+}=\emptyset$ and there is no 1 -SPP.

Theorem 2.3 - Countable infinity of $Z^{+}$. If $\omega_{1} / \omega_{2} \notin \mathbb{Q}$ then $Z$ is countably infinite. Moreover, if Assumption 2.1 holds, $Z^{+}$is also countably infinite.

The proof of this theorem is exposed in Section 6. It is straightforward to show that $Z$ is countably infinite when $\omega_{1} / \omega_{2} \notin \mathbb{Q}$ since $h(\cdot)$ is quasi-periodic with many vertical asymptotes. The challenging part in Theorem 2.3 is to prove that $Z^{+}$is also infinite. Incidentally, it turns out that $Z^{-}$is also infinite and more precisely that $\operatorname{card}\left(Z^{+} \cap[0 ; A]\right) \sim \operatorname{card}\left(Z^{-} \cap[0 ; A]\right)$ for large $A$. It means that many roots of $h$ do not correspond to 1-SPP. Not only a 1-SPP is a rare object but among the roots of the function $h$, only a few correspond to a admissible 1-SPP.

In the next Section, the procedure to find 1-SPP numerically is detailed.

\section{EXAMPLES}

To construct 1-SPP, Theorems 2.1 and 2.2 are interpreted as follows: let $s>0$ satisfy $h(s)=0$ and $w_{1}(s)>0$. Such $s$ is a candidate to construct a 1-SPP $\mathbf{u}$ of Problem (1.1) corresponding to the initial data $\left[u_{1}(0), u_{2}(0), \dot{u}_{1}(0), \dot{u}_{2}(0)\right]^{\top}=[d, d,+v, 0]^{\top}$ where $v=d / w_{1}(s)$ with a sticking phase on $[0 ; \tau]$ and then a free-flight on $[\tau ; \tau+s]$ with $\tau=\mathcal{T}(s)$; more precisely:

- sticking phase for $t \in[0 ; \tau]$ : mass 2 sticks to the wall and mass 1 behaves as a 1 -dof linear oscillator.

- free flight for $t \in] \tau ; \tau+s[$ : System (1.1a) is solved with following "initial" data at time $\tau=\mathcal{T}(s)$ :

$$
\left[u_{1}(\tau), u_{2}(\tau), \dot{u}_{1}(\tau), \dot{u}_{2}(\tau)\right]^{\top}=[d, d,-v, 0]^{\top} .
$$

The condition $u_{2}(t)<d$ is to be checked on the interval ] $\tau ; \tau+s$ [ to obtain a real solution of Problem (1.1). Otherwise, an impact emerges before $\tau+s$ and the assumption of a free flight is violated on $] \tau ; \tau+s$ [ so that the corresponding $\mathbf{u}(t)$ is not a 1-SPP.

Accordingly, building one 1-SPP requires two numerical steps:

(1) Compute the roots of $h(\cdot)$ : Figure 3 depicts the set of roots as the intersections of $h(\cdot)$ and the $x$-axis.

(2) Check the admissibility of the associated solution: check if $v>0$ and if $u_{2}(t)<d$ for all $t \in] \mathcal{T}(s) ; \mathcal{T}(s)+s[$. From the symmetry of the solution during the free flight, it is sufficient to check $u_{2}(t)<d$ for all $\left.t \in\right] \mathcal{T} ; \mathcal{T}+s / 2[$.

First numerical examples are provided with $m_{1}=m_{2}=1 \mathrm{~kg}, k_{1}=k_{2}=1 \mathrm{~N} / \mathrm{m}$. Hence, the two natural periods of the unconstrained linear System (1.1a) are $T_{1} \approx 10.17 \mathrm{~s}$ and $T_{2} \approx 3.88 \mathrm{~s}$.

Figure 2 shows the simplest 1-SPP one can find: only one loop for the orbit of the second mass. This orbit is very smooth except at one point corresponding to the whole sticking phase. At this sticking point, a $C^{1.5}$-regularity only is achieved as discussed in Section 4.3. Various examples featuring other responses are introduced in Figures 4 and 5. Many roots of $h$ belonging to $Z^{+}$do not correspond to 1-SPP. For 


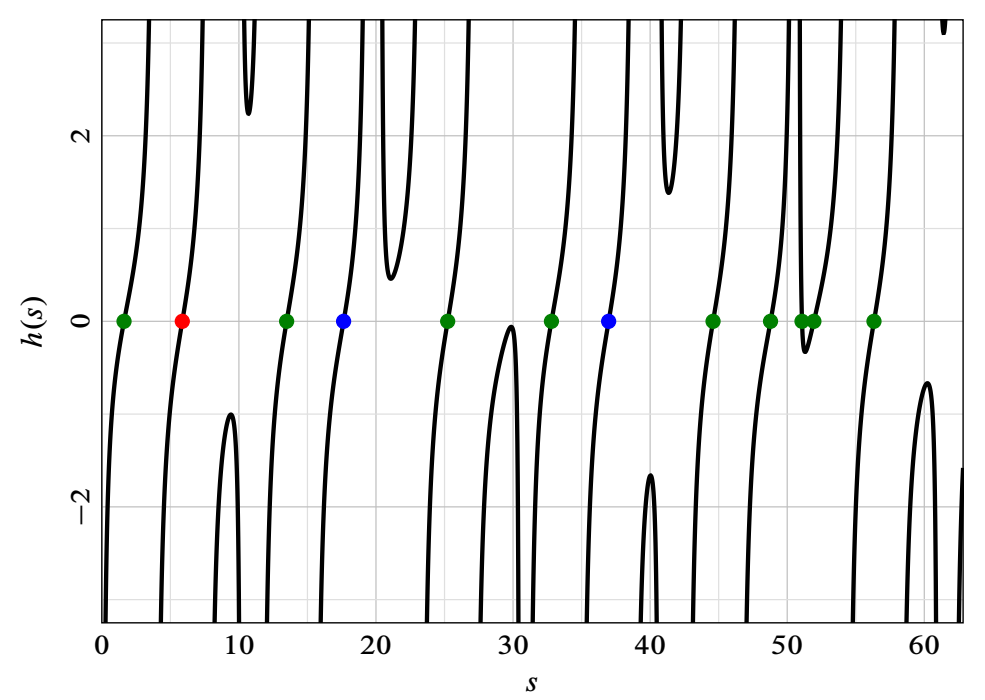

FIGURE 3. Roots $s \in Z=\{h(s)=0\}$ : red point: $s \in Z^{\text {ad }}$; blue points: $s \in Z^{+}$but $s \notin Z^{\text {ad }}$; green points: $s \in Z^{-}$. The set of $s$ corresponding to the admissible initial data are points in blue or red but only one point correspond to a 1-SPP: the red point.
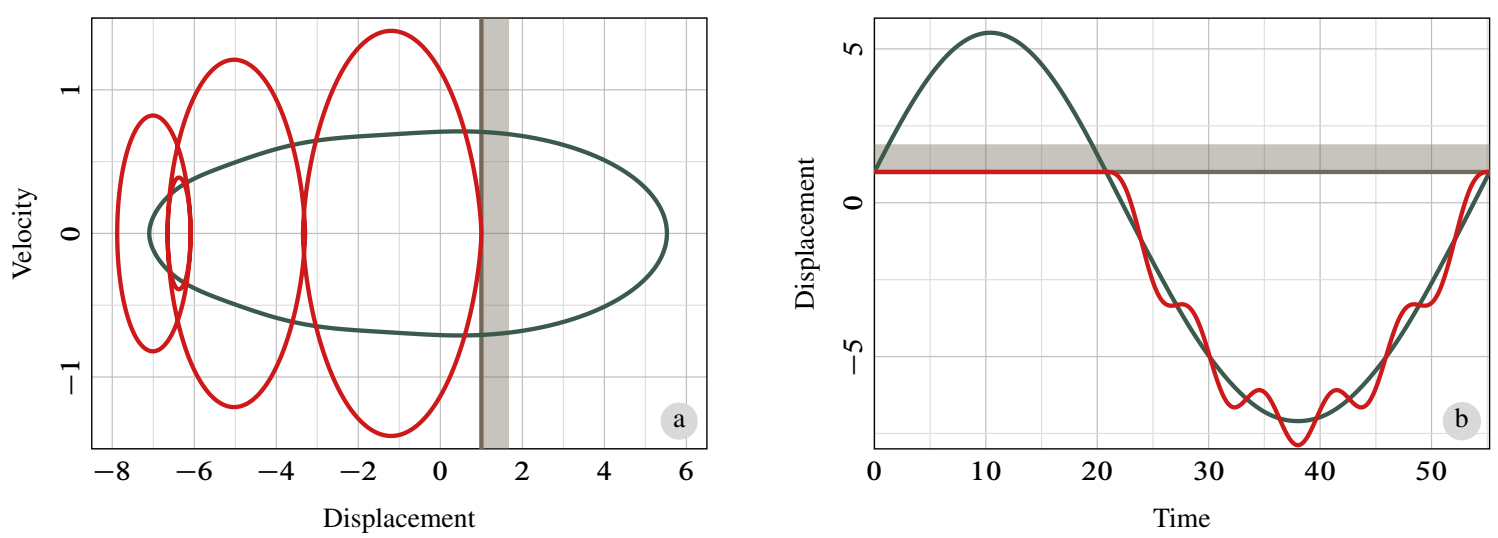

FIGURE 4. Admissible 1-SPP with $k_{1}=k_{2}=1 ; m_{1}=100 ; m_{2}=1$, initial data $U(0)=[1,1,0.7070682,0]: s \approx 34.412 \mathrm{~s}$ and $\mathcal{T} \approx 20.804$ s. (a) Orbits. (b) Displacements

instance, for $s \approx 17.97 \in Z^{+}$, the free-flight is not acceptable since the second mass penetrates the rigid obstacle, as pictured in Figure 6 . The condition $s \in Z^{+}$only stipulates that the non-penetration constraint (2.13) is satisfied near the sticking phase. Although $Z^{+}$is countably infinite, it is challenging to find the set $Z^{\text {ad }} \subset Z^{+}$yielding 1-SPP. At first, for large $s$, the free-flight lasts a long period of time and the possibility that $u_{2}$ exceeds $d$ seems to increase. Nevertheless, 1-SPP with large $s$ are founded in Figures 4-5.

\section{STICKING CONTACT}

This Section is devoted to the mathematical proof of Theorem 2.1 concerned with the necessary and sufficient conditions on the occurrence of a sticking phase ${ }^{1}$. The theory for such systems with impacts can be found in $[1,4,7,5,16]$.

In order to experience a sticking phase, the first necessary condition is a zero-velocity of the contacting mass when the gap is being closed, see below or $[4,7]$. Then, the sticking phase holds whenever there is a positive force generated from mass 1 on mass 2 . This force is explicit through the last equation of (1.1a). Next, the sticking system is presented. Via Lemma 4.1, it is clear that the energy of the unconstrained

\footnotetext{
${ }^{1}$ This Theorem was also stated in [9].
} 

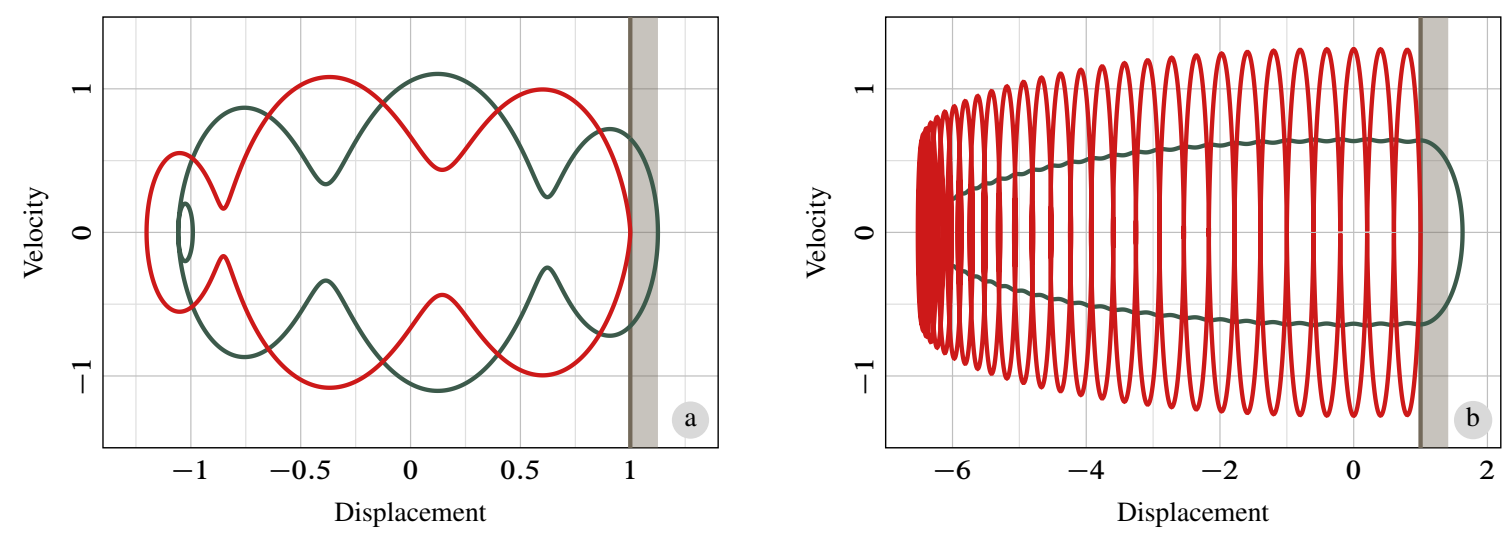

FIGURE 5. 1-SPP Orbits. (a) $k_{1}=1, k_{2}=10 k_{1}$ and $m_{1}=m_{2}=1$, initial data $U(0)=[1,1,0.6525913,0]$. (b) $k_{1}=1, k_{2}=100 k_{1}$ and $m_{2}=1, m_{1}=100 m_{2}$, initial data $U(0)=[1,1,0.6409175,0]$.
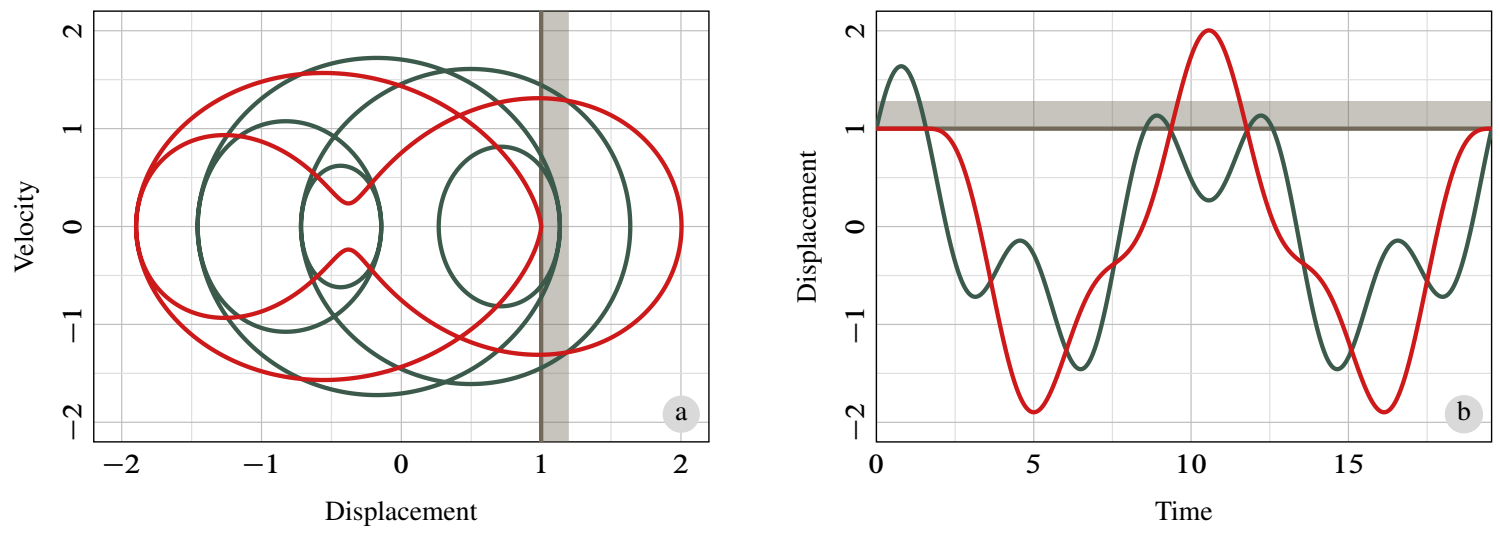

FIGURE 6. Non-admissible 1-SPP with initial data $U(0)=[1,1,1.4447006,0]$ : mass 2 penetrates the wall during the free flight. (a) Orbits. (b) Displacements.

linear free flight system is conserved by the sticking system which becomes simply a 1-dof problem: the closed-form solution as well as the explicit duration of sticking phase are obtained.

The solution is analytic away from the beginning and the end of the sticking phase [4]. It can be seen from Figure 2(c) that the contact force is only Lipschitz at the beginning and at the end of the sticking phase. Thus, $\ddot{u}_{2}$ is a Lipschitz function, so the function $u_{2}$ belongs to the Sobolev space $W^{3, \infty}$. The smoother function $u_{1}$ belongs to $C^{4} \cap W^{5, \infty}$ : for both functions $u_{1}$ and $u_{2}$, the singularity is located on the boundary of the sticking phase. The orbit $\left\{\left(u_{2}(t), \dot{u}_{2}(t)\right), t \in \mathbb{R}\right\}$ has only a $C^{1.5}$-regularity at the sticking point. The singularity $C^{1.5}$ is visible in Figure 2 and is caused by the zero velocity and zero acceleration of mass 2 exactly when the sticking phase starts and ends. This loss of regularity is explored at the end of this Section. The prestressed case $d \leq 0$ is covered in Section 7 with the occurrence of sticking phases of infinite duration.

4.1. Occurrence of a sticking phase. The proof for the necessary and sufficient conditions for a sticking phase to occur as stated in Theorem 2.1 is now given for $d>0$. The case $d<0$ is explained just after this proof.

Proof. The right and left analyticity of the solution for the perfect elastic rebound is used (Proposition 19 in [4]). Notice that the condition of a closed contact, i.e. $u_{2}(0)=d$, with zero velocity $\dot{u}_{2}^{-}(0)=0$ is mandatory. Otherwise $\dot{u}_{2}^{-}(0)>0, \dot{u}_{2}^{+}(0)=-\dot{u}_{2}^{-}(0)<0$ and the mass immediately leaves the wall, that is $u_{2}(t)<d$ for $t>0$ and $t \approx 0$ such that there is no sticking phase. The second equation of 
System (1.1a) is rewritten with the aforementioned initial data for mass 2 only:

$$
\begin{aligned}
& m_{2} \ddot{u}_{2}(t)=k_{2}\left(u_{1}(t)-u_{2}(t)\right)+R(t) \\
& u_{2}(0)=d, \quad \dot{u}_{2}^{-}(0)=0, \quad R(t) \leq 0
\end{aligned}
$$

During a sticking phase $u_{2}(t) \equiv d$ so $\ddot{u}_{2}(t) \equiv 0$ and Equation (4.1) yields the relation between the reaction $R(t)$ and the displacement $u_{1}(t)$ :

$$
R(t)=k_{2}\left(d-u_{1}(t)\right)
$$

which is non-positive if and only if

$$
u_{1}(t) \geq d
$$

Let us emphasize that Condition (4.3) is important in this work even though there is no unilateral constraint on $u_{1}$ in the formulation of System (1.1). During the sticking phase, Inequality (4.3) is satisfied. As a consequence, $\dot{u}_{2}(0)=0$ is not a sufficient condition to ensure the existence of a sticking phase starting at time $t=0$. Various situations depending on the state $\left(u_{1}(0), \dot{u}_{1}(0)\right)$ should be scrutinized:

$u_{1}(0)<d$ : The left-hand side of $(4.1)$ is strictly negative and so is $\ddot{u}_{2}^{+}(0)$. There is no sticking phase. More precisely, $u_{2}$ is a piecewise analytic function [4] and its Taylor series in the right neighbhourhood of 0 is:

$$
u_{2}(t)=d+t^{2} \frac{\ddot{u}_{2}^{+}(0)}{2}+O\left(t^{3}\right)<d
$$

$u_{1}(0)>d$ : Since $u_{1}$ is continuous, it remains larger than $d$ in a right neighbhourhood $\left[0 ; \varepsilon_{2}[\right.$ of $t=0$. Thus, there is a positive force $F(t)=k_{2}\left(u_{1}(t)-u_{2}(t)\right)$ acting on mass 2 , and by Newton's third law, there exists a reaction $R(t)$ such that $R(t)=-F(t)$. Substitution into (4.1) yields $\ddot{u}_{2}(t)=0$, $\forall t \in\left[0 ; \varepsilon_{2}\left[\right.\right.$. Hence $u_{2}(t)=d, \forall t \in\left[0 ; \varepsilon_{2}[\right.$, i.e. a sticking phase emerges.

$u_{1}(0)=d: \ddot{u}_{2}^{+}(0)=0$ and there are three possibilities for the velocity of mass 1 :

(1) If $\dot{u}_{1}(0)>0, u_{1}(t)$ becomes immediately larger than $d$ for $t>0$ small enough. This is similar to the previous case where a sticking phase occurs.

(2) If $\dot{u}_{1}(0)<0$ then $u_{1}(t)$ becomes immediately smaller than $d$ and no sticking phase occurs. More precisely from Equation (4.1), $m_{2} \dddot{u}_{2}^{+}(0)=k_{2}\left(\dot{u}_{1}(0)-\dot{u}_{2}(0)\right)<0$ and the Taylor series of $u_{2}$ in the right neighbhourhood of 0 is

$$
u_{2}(t)=u_{2}(0)+t \dot{u}_{2}(0)+\frac{t^{2}}{2} \ddot{u}_{2}^{+}(0)+\frac{t^{3}}{6} \dddot{u}_{2}^{+}(0)+O\left(t^{4}\right)=d+0+0+t^{3} \frac{\dddot{u}_{2}^{+}(0)}{6}+O\left(t^{4}\right)<d
$$

(3) If $\dot{u}_{1}(0)=0$, then $\ddot{u}_{1}^{+}(0)=-k_{1} d / m_{1}<0$. Thus $m_{2} \dddot{u}_{2}^{+}(0)=k_{2}\left(\dot{u}_{1}(0)-\dot{u}_{2}(0)\right)=0$ and $m_{2} u_{2}^{(4)+}(0)=k_{2}\left(\ddot{u}_{1}^{+}(0)-\ddot{u}_{2}^{+}(0)\right)<0$. Similarly, a Taylor series of $u_{2}$ in the right neighbourhood of 0 shows that $u_{2}(t)<d$ for $t>0$ and $t \approx 0$. Thus, there is no sticking phase.

Note that only the last case $u_{1}(0)=d$ and $\dot{u}_{1}(0)=0$ crucially depends on the sign of $d$. It is further discussed in Section 7 when $d \leq 0$. Moreover, all piecewise analytic solutions presented above preserve energy. It is clear for the grazing case since $R \equiv 0$. When sticking occurs, energy conservation is a consequence of Lemma 4.1. In conclusion, every introduced case corresponds to the unique solution preserving energy [4].

Remark 4.1. The duration of the sticking phase directly relates to the reaction $R(t)$. The sign of $R(t)$ is given by the sign of $d-u_{1}(t)$ via (4.2). Thus, for $d<0$, an infinite sticking phase appears when the solution $u_{1}$ of the sticking equation (4.6) satisfies $u_{1}(t) \geq d=-|d|$ for all time, which is possible as detailed in Section 7.

4.2. Sticking system. It is now shown that the solution with a sticking phase expounded in Section 4.1 is the unique solution which preserves the total energy, see [4].

The notion of sticking system is explained in [7]. It is the reduced system during the sticking phase. Since the last mass is at rest, the system loses one degree of freedom. From the previous developments, 
the sticking system complemented by the initial data at the beginning of a sticking phase is explicitly derived as

$$
\begin{array}{rll}
m_{1} \ddot{u}_{1}+\left(k_{1}+k_{2}\right) u_{1}-k_{2} u_{2}=0, & u_{1}(0)=d, & \dot{u}_{1}(0)=v>0, \\
m_{2} \ddot{u}_{2}=0, & u_{2}(0)=d, & \dot{u}_{2}(0)=0 .
\end{array}
$$

The sticking system (4.4), (4.5) becomes simply a sticking equation (4.6). The initial data for mass 1 has to be clarified. If $u_{1}(0)>d$ then this inequality is also valid locally in the past, and the sticking phase exists before $t=0$. If $u_{1}(0)=d$ and $\dot{u}_{1}(0)>0$, then there exists $\eta>0$ such that $u_{1}(t)<d$ for $-\eta<t<0$ so there is no sticking phase just before $t=0$, in other words, $t=0$ is the beginning of the sticking phase. The grazing contact case $\dot{u}_{1}(0)=0$ and the case where constraint (2.13) is violated, $\dot{u}_{1}(0)<0$, do not have to be considered.

During free-flight, there is a total energy (1.1d) for the symmetric system (2.12). However, during the sticking phase, system (4.4)-(4.5) is not symmetric. Accordingly, the question of conservation of energy during the sticking phase is not obvious. It is proven in the next lemma.

Lemma 4.1. The solution to Equations (4.4) and (4.5) conserves the energy $\mathbf{E}$ (1.1d).

Proof. Assume that $t=0$ is the beginning of a sticking phase and $t=\tau$, the end. During this sticking phase on the interval $[0 ; \tau]$, the governing equations are

$$
\begin{aligned}
& m_{1} \ddot{u}_{1}+\left(k_{1}+k_{2}\right) u_{1}=k_{2} d, \\
& u_{2}=d .
\end{aligned}
$$

The first equation conserves the energy around the new equilibrium $\bar{u}_{1}=k_{2} d /\left(k_{1}+k_{2}\right)$ :

$$
E_{1}(t)=m_{1} \dot{u}_{1}^{2}(t)+\left(k_{1}+k_{2}\right)\left(u_{1}(t)-\bar{u}_{1}\right)^{2}=E_{1}(0) .
$$

Moreover, since $u_{1}(t)=\left(u_{1}(t)-\bar{u}_{1}\right)+\bar{u}_{1}$ and $u_{2}(t)=d$, an easy computation yields:

$$
\left(k_{1}+k_{2}\right) u_{1}^{2}(t)=\left(k_{1}+k_{2}\right)\left(u_{1}(t)-\bar{u}_{1}\right)^{2}+2 k_{2} u_{1}(t) u_{2}(t)+C
$$

and $C=-\left(k_{1}+k_{2}\right) \bar{u}_{1}^{2}$. The energy of System (1.1) can be calculated. Since $\dot{\mathbf{u}}$ is continuous along a sticking phase, the exponents \pm are dropped.

$$
\begin{aligned}
\mathbf{E}(t) & =\dot{\mathbf{u}}^{\top}(t) \mathbf{M} \dot{\mathbf{u}}(t)+\mathbf{u}^{\top}(t) \mathbf{K u}(t) \\
& =m_{1} \dot{u}_{1}^{2}(t)+\left(k_{1}+k_{2}\right) u_{1}^{2}(t)+m_{2} \dot{u}_{2}^{2}(t)+k_{2} u_{2}^{2}(t)-2 k_{2} u_{1}(t) u_{2}(t) \\
& =m_{1} \dot{u}_{1}^{2}(t)+\left(k_{1}+k_{2}\right)\left(u_{1}(t)-\bar{u}_{1}\right)^{2}+2 k_{2} u_{1}(t) u_{2}(t)+C+0+k_{2} d^{2}-2 k_{2} u_{1}(t) u_{2}(t) \\
& =E_{1}(t)+C+k_{2} d^{2}=E_{1}(0)+C+k_{2} d^{2}=\mathbf{E}(0) .
\end{aligned}
$$

This ends the proof: the total energy of the system is constant for all 1-SPP.

The sticking system is now solved and the sticking time is explicitly exhibited: this is an interesting feature of our 2-dof mechanical system. The 1-dof linear oscillator problem with a constant force (4.6) has the explicit solution

$$
u_{1}(t)=A \cos (\omega t+\phi)+\frac{k_{2}}{k_{1}+k_{2}} d \quad \text { where } \quad \omega=\sqrt{\frac{k_{1}+k_{2}}{m_{1}}} .
$$

The expression of the constants $A$ and $\phi$ stems from the initial condition $\left[u_{1}(0), \dot{u}_{1}(0)\right]^{\top}=[d, v]^{\top}$ as follows

$$
A=\frac{k_{1} d}{\left(k_{1}+k_{2}\right) \cos (\phi)} \quad \text { and } \quad \phi=-\arctan (\xi v) \quad \text { with } \quad \xi=\frac{\sqrt{\left(k_{1}+k_{2}\right) m_{1}}}{k_{1} d}
$$

and $\mathcal{T}$ is the first positive time satisfying $u_{1}(\mathcal{T})=d$, that is $\mathcal{T}=2 \arctan (\xi v) / \omega$. Due to the symmetry of the solution to the 1-dof Problem (4.6) with respect to the $u_{1}$ axis in the plane $\left(u_{1}, \dot{u}_{1}\right), u_{1}(\mathcal{T})=d$ and $\dot{u}_{1}(\mathcal{T})=-v$ which also means that $\mathcal{T}$ is the end of the sticking phase via Theorem 2.1. 
4.3. 1.5-singularity at the sticking point. The following Proposition states precisely the regularity near a sticking phase, essentially $C^{2}$ and almost $C^{3}$. The lower $C^{1.5}$-regularity of the orbit is obtained at the end of the Section.

Proposition 4.2 - Regularity of solutions. Assume $\mathbf{u}(\cdot)$ is a solution of System (1.1) on $\left[T_{0} ; T_{1}\right]$ with only a sticking phase on $[0 ; \mathcal{T}]$ and a free flight elsewhere with $T_{0}<0<\mathcal{T}<T_{1}$. Then $u_{1} \in C^{4}\left(\left[T_{0} ; T_{1}\right]\right) \cap W^{5, \infty}\left(\left[T_{0} ; T_{1}\right]\right)$ and $u_{2} \in C^{2}\left(\left[T_{0} ; T_{1}\right]\right) \cap W^{3, \infty}\left(\left[T_{0} ; T_{1}\right]\right)$.

Proof. Away from the strict beginning and end of the sticking phase, the solution is regular: analytic outside $[0 ; \mathcal{T}], u_{1}$ is analytic and $u_{2}$ is constant inside $] 0 ; \mathcal{T}$. The solution regularity at $t=0$ and $t=\mathcal{T}$ is of higher interest. Only the case $t=0$ is considered since the other case $t=\mathcal{T}$ is quite similar. The initial data at $t=0$ is $\left[\mathbf{u}(0)^{\top}, \dot{\mathbf{u}}(0)^{\top}\right]^{\top}=[d, d, v, 0]^{\top}$. The second Equation within (1.1a) is

$$
m_{2} \ddot{u}_{2}(t)=k_{2}\left(u_{1}(t)-u_{2}(t)\right)+R(t) .
$$

During the sticking phase, $0<t<\mathcal{T}, u_{2}(t)=d$ so $\ddot{u}_{2}(t)=0$ and $\ddot{u}_{2}^{+}(0)=0$ and before the sticking phase, $t<0$, since $u_{2}(t)<d, R(t)=0$ and $\lim _{0>t \rightarrow 0} u_{2}(t)=d=\lim _{0>t \rightarrow 0} u_{1}(t)$ so from Equation (4.8) $\ddot{u}_{2}^{-}(0)=0$, thus $\ddot{u}_{2}$ is continuous at time $t=0$ and $\ddot{u}_{2}(0)=0$. However, the third derivative of $u_{2}$ on the left of $t=0$ does not vanish since $m_{2} \dddot{u}_{2}^{-}(0)=k_{2}\left(\dot{u}_{1}(0)-\dot{u}_{2}(0)\right)=k_{2} v>0$ and $\dddot{u}_{2}$ is then bounded. Hence, $u_{2} \in C^{2}([0 ; T]) \cap W^{3, \infty}([0 ; T])$.

The regularity of $u_{1}$ is investigated from the first Equation of (1.1a) which reads

$$
m_{1} \ddot{u}_{1}+\left(k_{1}+k_{2}\right) u_{1}=k_{2} u_{2}
$$

and shows that $\ddot{u}_{1}$ and $u_{2}$ have the same regularity. Accordingly, $\ddot{u}_{1} \in C^{2}([0 ; T]) \cap W^{3, \infty}([0 ; T])$ that is $u_{1} \in C^{4}([0 ; T]) \cap W^{5, \infty}([0 ; T])$.

We now prove the $C^{1.5}$-regularity of the orbit without using explicit formula.

Proof. The $C^{1.5}$-smoothness of the projection of the orbit on the last component, more precisely the regularity of the set

$$
\Gamma_{2}=\left\{\gamma(t)=\left(u_{2}(t), \dot{u}_{2}(t)\right), 0 \leq t \leq T\right\} \subset \mathbb{R}^{2}
$$

is explored. By $T$-periodicity, this parametrization is defined for all time. During the sticking phase, the last mass rests against the foundation, $\gamma(t)=\gamma(0)=(d, 0), \dot{\gamma}(t)=(0,0)$ for $0 \leq t \leq \mathcal{T}$ and the parametrization is then singular. Instead, a regular parametrization of $\Gamma_{2}$ is thus proposed in the form $\tilde{\gamma}(t)=\gamma(t-\mathcal{T}), \mathcal{T} \leq t \leq T$. In other words, $\tilde{\gamma}$ is $\gamma$ where the sticking phase has been removed. Also, $\tilde{\gamma}$ is defined for all time through $s$-periodicity with $s=T-\mathcal{T}$. The set $\tilde{\Gamma}_{2}=\tilde{\gamma}([0 ; s])$ is exactly $\Gamma_{2}$. The curve is analytic away from the sticking point $(d, 0)$. A precise study of $\tilde{\gamma}(t),|t|<\varepsilon$ should now be undertaken for $\varepsilon>0$ sufficiently small. To this end, the left and right derivatives are computed since the solution is left and right analytic at the sticking point [4]:

$$
\frac{\mathrm{d}^{k}}{\mathrm{~d} t^{k}} \tilde{\gamma}^{-}(0)=\frac{\mathrm{d}^{k}}{\mathrm{~d} t^{k}} \gamma^{-}(0), \quad \frac{\mathrm{d}^{k}}{\mathrm{~d} t^{k}} \tilde{\gamma}^{+}(0)=\frac{\mathrm{d}^{k}}{\mathrm{~d} t^{k}} \gamma^{+}(\mathcal{T})
$$

To compute the successive left and right derivatives, the ODE

$$
m_{2} \ddot{u}_{2}(t)=k_{2}\left(u_{1}(t)-u_{2}(t)\right)
$$

is used just before the sticking phase and just after the sticking phase. Recall that $u_{1}(0)=d$ and $\dot{u}_{1}(0)=v>0, u_{2}(0)=d$ and $\dot{u}_{2}(0)=0, u_{1}(\mathcal{T})=d$ and $\dot{u}_{1}(\mathcal{T})=-v<0, u_{2}(\mathcal{T})=d$ and $\dot{u}_{2}(\mathcal{T})=0$. The ODE gives $m_{2} \ddot{u}_{2}^{-}(0)=k_{2}(d-d)=0, \ddot{u}_{2}^{+}(\mathcal{T})=0$, so $\dot{\tilde{\gamma}}^{ \pm}(0)=(0,0)$. The parametrization is still singular and higher derivatives of $u_{2}$ are computed by differentiating the ODE, that is

$$
\begin{aligned}
& m_{2} \dddot{u}_{2}(t)=k_{2}\left(\dot{u}_{1}(t)-\dot{u}_{2}(t)\right) \\
& \ddot{\tilde{\gamma}}^{-}(0)=(0, \beta), \quad \ddot{\tilde{\gamma}}^{+}(0)=(0,-\beta), \quad \beta=k_{2} v / m_{2}>0 \\
& m_{2} \dddot{u}_{2}(t)=k_{2}\left(\ddot{u}_{1}(t)-\ddot{u}_{2}(t)\right) \\
& \ddot{\tilde{\gamma}}^{-}(0)=(\beta, \delta), \quad \ddot{\tilde{\gamma}}^{+}(0)=(-\beta, \delta), \quad \delta=-k_{2} k_{1} d /\left(m_{2} m_{1}\right)<0
\end{aligned}
$$


where the second derivative of $u_{1}$ comes from the equation $m_{1} \ddot{u}_{1}(t)=-k_{1} u_{1}(t)-k_{2}\left(u_{1}(t)-u_{2}(t)\right)$ : $\ddot{u}_{1}(0)=-k_{1} d / m_{1}<0$. The local behaviour at $t=0$ is then for $\pm t>0$ :

$$
\tilde{\gamma}(t)=(d, 0)+\frac{1}{2} \operatorname{sign}(t)(0, \beta) t^{2}+\frac{1}{6}(\operatorname{sign}(t) \beta, \delta) t^{3}+\mathcal{O}\left(t^{4}\right) .
$$

There are two singularities for this parametrization: the left and right expansions for $\pm t>0$, and the more important $\dot{\tilde{\gamma}}(0)=(0,0)$. To clearly identify the regularity of the curve at $t=0$, a last change of variable is performed [15]: $\tau=\operatorname{sign}(t) t^{2}$ and $\hat{\gamma}(\tau)=\tilde{\gamma}(t)$ such that:

$$
\hat{\gamma}(\tau)=(d, 0)+\frac{1}{2}(0, \beta) \tau+\frac{1}{6}(\beta, \operatorname{sign}(\tau) \delta)|\tau|^{1.5}+\mathcal{O}\left(\tau^{2}\right) .
$$

The $C^{1.5}$-regularity is then identified since $\dot{\hat{\gamma}}(0) \neq(0,0)$ and this is optimal.

\section{BUILDING 1-SPP}

This Section addresses the construction of the 1-SPP developed in Section 2.2. An explicit formula for $\mathcal{T}$ is obtained and the set of admissible initial data is derived. The initial velocity of the first mass depends on the free flight time $s$ and it is proven that $s$ can be found in the infinite set of roots of $h(\cdot)$. The symmetry of the solutions is also discussed.

5.1. Initial data. Without loss of generality, the initial data is defined in the Poincaré section $u_{2}=d$. The problem is to find a periodic function $\mathbf{u}$ generated by the initial data $[d, d, v, 0]^{\top}$ such that there is one sticking phase per period. As explained previously, $T$ and $\mathcal{T}$ are parametrized by $s$. The sticking solution and the sticking time $\mathcal{T}>0$ are calculated explicitly in Section 4.2.

By denoting $\mathbf{U}=\left[\mathbf{u}^{\top}, \dot{\mathbf{u}}^{\top}\right]^{\top}$, a free flight starts with the following initial data at time $\mathcal{T}$ :

$$
\mathbf{U}(\mathcal{T})=[d, d,-v, 0]^{\top} .
$$

It can be written as

$$
\mathbf{U}(\mathcal{T})=\mathbf{S U}(0) \quad \text { where } \quad \mathbf{S}=\left[\begin{array}{ll}
\mathbf{I} & \mathbf{0} \\
\mathbf{0} & \mathbf{L}
\end{array}\right] \text { and } \mathbf{L}=\left[\begin{array}{cc}
-1 & 0 \\
0 & 1
\end{array}\right] .
$$

Away from the sticking phase, system (1.1a) simplifies to

$$
\mathbf{M u ̈}+\mathbf{K u}=\mathbf{0} .
$$

Through the change of variable $\mathbf{u}=\mathbf{P q}$, Equation (5.3) becomes

$$
\mathbf{I} \ddot{\mathbf{q}}+\boldsymbol{\Omega}^{2} \mathbf{q}=\mathbf{0}
$$

which features the following block matrix solution

$$
\left.\mathbf{Q}(t)=\left[\begin{array}{c}
\mathbf{q}(t) \\
\dot{\mathbf{q}}(t)
\end{array}\right]=\mathbf{R}(t-\mathcal{T})\left[\begin{array}{l}
\mathbf{q}(\mathcal{T}) \\
\dot{\mathbf{q}}(\mathcal{T})
\end{array}\right], \quad \forall t \in\right] \mathcal{T} ; T[
$$

where

$$
\mathbf{R}(t)=\left[\begin{array}{cc}
\cos (t \Omega) & \boldsymbol{\Omega}^{-1} \sin (t \Omega) \\
-\boldsymbol{\Omega} \sin (t \Omega) & \cos (t \boldsymbol{\Omega})
\end{array}\right] .
$$

We shall find the solution $\mathbf{u}$ and the period $T$ such that

$$
\mathbf{Q}(T)=\mathbf{Q}(0) \text {. }
$$

By denoting $s=T-\mathcal{T}$, Equation (5.7) projected onto modal coordinates reads

$$
\mathbf{R}(s) \tilde{\mathbf{S}} \mathbf{Q}(0)=\mathbf{Q}(0) \quad \text { where } \quad \tilde{\mathbf{S}}=[\mathbf{B}] \mathbf{S}[\mathbf{P}] \quad \text { and } \quad[\mathbf{P}]=\left[\begin{array}{ll}
\mathbf{P} & \mathbf{0} \\
\mathbf{0} & \mathbf{P}
\end{array}\right]
$$

which can be expressed as $(\mathbf{R}(s) \tilde{\mathbf{S}}-\mathbf{I}) \mathbf{Q}(0)=\mathbf{0}$ with

$$
\mathbf{R}(s) \tilde{\mathbf{S}}-\mathbf{I}=\left[\begin{array}{cc}
\cos (\boldsymbol{\Omega} s)-\mathbf{I} & \boldsymbol{\Omega}^{-1} \sin (\boldsymbol{\Omega} s) \mathbf{B L P} \\
-\boldsymbol{\Omega} \sin (\boldsymbol{\Omega} s) & \cos (\boldsymbol{\Omega} s) \mathbf{B L P}-\mathbf{I}
\end{array}\right]
$$

The computations are similar to those introduced in [11]. This similarity will be explained later through the relationship between 1-SPP and the one-Impact-Per-Period solutions (1-IPP) detailed in [11 $]^{2}$.

\footnotetext{
${ }^{2}$ Note that there is a change of sign in $\mathbf{w}(s)$ due to the coefficient $a_{k j}=-P_{k j} B_{j 1}$ instead of $P_{k j} B_{j N}$ in [11].
} 
The duration $s$ is assumed not to be a period of the linear differential system, $s \notin \cup_{j=1}^{2} T_{j} \mathbb{Z}$ where $T_{j}=2 \pi / \omega_{j}, j=1,2$ are frequencies of the linear system. Then, the following quantities are well defined:

$$
\begin{aligned}
& (s)=(\mathbf{I}-\cos (\boldsymbol{\Omega} s))^{-1} \boldsymbol{\Omega}^{-1} \sin (\boldsymbol{\Omega} s), \\
& \mathbf{w}(s)=\mathbf{P}(s) \mathbf{B L} \mathbf{e}_{1}, \quad \mathbf{e}_{1}=(1,0)^{\top}, \\
& w_{1}(s)=\mathbf{e}_{1}^{\top} \mathbf{w}(s) .
\end{aligned}
$$

The solution set of initial data yielding 1-SPP, possibly "ghost" solutions if constraint (2.13) is violated, is described explicitly via the following lemma:

Lemma 5.1. If $s \notin \cup_{j=1}^{2} T_{j} \mathbb{Z}$ then the system

$$
\mathbf{R}(s) \tilde{\mathbf{S}} \mathbf{Q}(0)=\mathbf{Q}(0)
$$

defines a one dimensional vector space parametrized by $c \in \mathbb{R}$ given in variables

$$
\left[\begin{array}{c}
\mathbf{u}(0) \\
\dot{\mathbf{u}}(0)
\end{array}\right]=\mathbf{P}\left[\begin{array}{c}
\mathbf{q}(0) \\
\dot{\mathbf{q}}(0)
\end{array}\right]=c\left[\begin{array}{c}
\mathbf{w}(s) \\
\mathbf{e}_{1}
\end{array}\right]
$$

Proof. Compute $\operatorname{ker}(\mathbf{R}(s) \tilde{\mathbf{S}}-\mathbf{I})$ by blocks (see [11]):

$$
\left[\begin{array}{cc}
\cos (\boldsymbol{\Omega} s)-\mathbf{I} & \boldsymbol{\Omega}^{-1} \sin (\boldsymbol{\Omega} s) \mathbf{B L P} \\
-\boldsymbol{\Omega} \sin (\boldsymbol{\Omega} s) & \cos (\boldsymbol{\Omega} s) \mathbf{B L P}-\mathbf{I}
\end{array}\right] \sim\left[\begin{array}{cc}
\cos (\boldsymbol{\Omega} s)-\mathbf{I} & \boldsymbol{\Omega}^{-1} \sin (\boldsymbol{\Omega} s) \mathbf{B L P} \\
\mathbf{0} & (\mathbf{L}+\mathbf{I}) \mathbf{P}
\end{array}\right]
$$

because the matrix $(\mathbf{I}-\cos (\boldsymbol{\Omega} s))^{-1} \mathbf{B}$ is invertible. Since $\dot{\mathbf{u}}=\mathbf{P} \dot{\mathbf{q}}$, the right lower block in (5.13) simplifies to $(\mathbf{L}+\mathbf{I}) \dot{\mathbf{u}}=\mathbf{0}$, that is $\dot{\mathbf{u}}=c \mathbf{e}_{1}$ with $c \in \mathbb{R}$. Similarly, the upper block provides the expression $\mathbf{q}=c(\mathbf{I}-\cos (\boldsymbol{\Omega} s))^{-1} \boldsymbol{\Omega}^{-1} \sin (\boldsymbol{\Omega} s) \mathbf{B L} \mathbf{e}_{1}$.

The assumption of lemma 5.1 is always valid and proven in the next lemma: the free flight duration of any 1-SPP is never a (multiple of a) period of the linear system.

Lemma 5.2. For a 1-SPP, the duration $s$ of the free-flight is not a linear period: $s \notin \cup_{j=1}^{2} T_{j} \mathbb{Z}$.

Proof. It is proven that there is no 1-SPP involving a free-flight duration as a linear period. In other words, if $s \in \cup_{j=1}^{2} T_{j} \mathbb{Z}$, i.e. there exists $k, \ell \in \mathbb{Z}$ such that $s=k T_{1}$ or $s=\ell T_{2}$, then the corresponding solutions must be linear grazing modes.

Firstly, let us emphasize that all the components of the matrix $\mathbf{P}=\left(P_{i j}\right)_{i, j=1}^{2}$ are nonzero in our case of study, a chain of two masses. The matrix $\mathbf{P}$ of eigenvectors can be computed explicitly and has the following formula

$$
\mathbf{P}=\left[\begin{array}{cc}
\frac{a+\sqrt{a^{2}+b^{2}}}{\sqrt{m_{1}\left(\left(a+\sqrt{a^{2}+b^{2}}\right)^{2}+b^{2}\right)}} & \frac{a-\sqrt{a^{2}+b^{2}}}{\sqrt{m_{1}\left(\left(a-\sqrt{a^{2}+b^{2}}\right)^{2}+b^{2}\right)}} \\
\frac{b}{\sqrt{m_{2}\left(\left(a+\sqrt{a^{2}+b^{2}}\right)^{2}+b^{2}\right)}} & \frac{b}{\sqrt{m_{2}\left(\left(a-\sqrt{a^{2}+b^{2}}\right)^{2}+b^{2}\right)}}
\end{array}\right]
$$

where

$$
a=\frac{1}{2}\left(\frac{k_{2}}{m_{2}}-\frac{k_{1}+k_{2}}{m_{1}}\right), \quad b=\frac{k_{2}}{\sqrt{m_{1} m_{2}}} .
$$

Hence, $P_{i j} \neq 0$ for all $i, j=1,2$.

Secondly, one shows that if $s=k T_{1}$ then the periodic solutions with the free-flight duration $s$ must be the first linear grazing mode. Consider $s=k T_{1}$, then the matrix $\mathbf{R}(s) \tilde{\mathbf{S}}-\mathbf{I}$ becomes

$$
\left[\begin{array}{cccc}
0 & 0 & 0 & 0 \\
0 & C_{2}-1 & S_{2} / \omega_{2} A_{21} & S_{2} / \omega_{2} A_{22} \\
0 & 0 & A_{11}-1 & A_{12} \\
0 & -\omega_{2} S_{2} & C_{2} A_{21} & C_{2} A_{22}-1
\end{array}\right]
$$


where $C_{2}=\cos \left(2 \pi \omega_{2} / \omega_{1}\right), S_{2}=\sin \left(2 \pi \omega_{2} / \omega_{1}\right)$, and $\mathbf{A}=\mathbf{B L P}$. With the assumption of no internal resonance, $C_{2} \neq 0, C_{2}-1 \neq 0$ and $S_{2} \neq 0$. Hence, after simple calculations, the last three equations become

$$
\left[\begin{array}{ccc}
C_{2}-1 & S_{2} / \omega_{2} A_{21} & S_{2} / \omega_{2} A_{22} \\
0 & A_{11}-1 & A_{12} \\
-\omega_{2} S_{2} & C_{2} A_{21} & C_{2} A_{22}-1
\end{array}\right] \sim\left[\begin{array}{ccc}
C_{2}-1 & S_{2} / \omega_{2} A_{21} & S_{2} / \omega_{2} A_{22} \\
0 & A_{11}-1 & A_{12} \\
0 & A_{21} & A_{22}+1
\end{array}\right]
$$

The last two equations can be rewritten as $(\mathbf{A}+\mathbf{L}) \dot{\mathbf{q}}(0)=\mathbf{0}$ or $(\mathbf{L P}+\mathbf{P L}) \dot{\mathbf{q}}(0)=\mathbf{0}$. Since $\mathbf{L P}+\mathbf{P L}=$ $\operatorname{diag}\left(-2 P_{11}, 2 P_{22}\right)$, where $P_{11}$ and $P_{22}$ are nonzero, this yields $\dot{\mathbf{q}}(0)=\mathbf{0}$. By substituting into the second equation, it follows that $q_{2}(0)=0$ or $u_{1}(0)=-B_{22} / B_{21} d$. Therefore, the initial data is $\left[u_{1}(0), d, 0,0\right]$ which corresponds to the first linear grazing mode.

Similarly, if $s=\ell T_{2}$ then the periodic solutions with the free-flight duration $s$ must be the second linear grazing mode. One obtains a similar expression $(\mathbf{A}-\mathbf{L}) \dot{\mathbf{q}}(0)=\mathbf{0}$ or $(\mathbf{L P}-\mathbf{P L}) \dot{\mathbf{q}}(0)=\mathbf{0}$. Since

$$
\mathbf{L P}-\mathbf{P L}=\left[\begin{array}{cc}
0 & -2 P_{12} \\
-2 P_{21} & 0
\end{array}\right]
$$

where both $P_{12}$ and $P_{21}$ are both non-vanishing terms, then $\dot{\mathbf{q}}(0)=\mathbf{0}$. Consequently, the initial data obtained correspond to the second linear grazing mode.

The parameter $c=\dot{u}_{1}^{+}(0)=v$ is identified from the third row of (5.12). By expressing the initial condition

$$
\left[\begin{array}{l}
u_{1}(0) \\
u_{2}(0)
\end{array}\right]=\left[\begin{array}{l}
d \\
d
\end{array}\right]=v\left[\begin{array}{l}
w_{1}(s) \\
w_{2}(s)
\end{array}\right]=c \mathbf{P}(s) \mathbf{B L e}_{1}
$$

System (5.14) simplifies to $w_{1}(s)=w_{2}(s)$ or

$$
h(s)=w_{1}(s)-w_{2}(s)=\sum_{j=1}^{2} \alpha_{j} \cot \left(\frac{\omega_{j} s}{2}\right)=0
$$

and the initial velocity of the first mass is found from

$$
v=\frac{d}{w_{1}(s)}, \quad w_{1}(s)>0 .
$$

If $\omega_{1} / \omega_{2} \notin \mathbb{Q}$, the function $h(\cdot)$ exhibits a countably infinite set of roots. Moreover the set of $s$ such that $h(s)=0$ and $v(s)>0$ is also countably infinite by Theorem 2.3. The particular case when $\omega_{1} / \omega_{2} \in \mathbb{Q}$ is discussed in Section 6.

\subsection{Symmetry. To conclude the validation of Theorem 2.2, the symmetry of 1-SPP is proven.}

Proof. Through periodicity, it is sufficient to check the symmetry of the solutions on one period only. The symmetry is satisfied during the sticking phase and the free flight. Since only the first mass oscillates during the sticking phase, the solution is symmetric. Let us check the symmetry of solutions during the free flight time $t \in[\mathcal{T} ; T]$ where $\mathbf{u}(\mathcal{T})=\mathbf{u}(T)$ and $\dot{\mathbf{u}}^{+}(\mathcal{T})=-\dot{\mathbf{u}}^{-}(T)$. Denoting $\theta=(T+\mathcal{T}) / 2$, it is sufficient to show that $\mathbf{u}(\theta+t)=\mathbf{u}(\theta-t), \forall t \in I=[-s / 2 ; s / 2]$. Let $\mathbf{z}_{+}$be the function defined on $I$ such that $\mathbf{z}_{+}(t)=\mathbf{u}(\theta+t)$. Then $\mathbf{z}_{+}$is a well defined smooth function on $I$ with $\mathbf{z}_{+}(s / 2)=\mathbf{u}(T)$ and $\dot{\mathbf{z}}_{+}(s / 2)=\dot{\mathbf{u}}^{-}(T)$. Similarly, by defining the function $\mathbf{z}_{-}(t):=\mathbf{u}(\theta-t)$, for $t \in I$, it can be checked that $\mathbf{z}_{-}(s / 2)=\mathbf{u}(\mathcal{T})$ and $\dot{\mathbf{z}}_{-}(s / 2)=-\dot{\mathbf{u}}^{+}(\mathcal{T})$. Furthermore, both $\mathbf{z}_{+}$and $\mathbf{z}_{-}$are solutions to the linear differential system $\mathbf{M} \ddot{\mathbf{z}}+\mathbf{K z}=\mathbf{0}$ on $I$. Notice that $\mathbf{z}_{+}$and $\mathbf{z}_{-}$have the same initial data $\mathbf{z}_{+}(s / 2)=\mathbf{z}_{-}(s / 2)$ and $\dot{\mathbf{z}}_{+}(s / 2)=\dot{\mathbf{z}}_{-}(s / 2)$. Hence, by the uniqueness of the initial value problem, it is deduced that $\mathbf{z}_{+}(t) \equiv \mathbf{z}_{-}(t)$ on $I$. This completes the proof on the symmetry of solutions.

5.3. Relationship between 1-SPP and 1-IPP. For this two-degree-of-freedom vibro-impact system, a relationship between one-sticking-phase-per-period and one-impact-per-period solutions [11] is exhibited. It clarifies the similarities and differences of such periodic solutions.

Consider the two Figures 2(a) and 2(b) showing a single loop in $\Gamma_{2}$. Figures 7 (a) and 7(b) are then obtained by "deleting" the sticking phase on the whole interval $] 0 ; \mathcal{T}$ [ such that a 1-IPP solution $[9,11]$ is identified, where the jump occurs on mass 1 (instead of mass 2) when $u_{1}(0)=d$ as well as $u_{2}(0)=d$. Take note that $u_{1}(t) \leq d$ for all time. It is important to note that this 1-IPP is "unique" is the sense that it satisfies $u_{2}(0)=d$; it is denoted 1-IPP in the remainder. The correspondence between 1-SPP and 

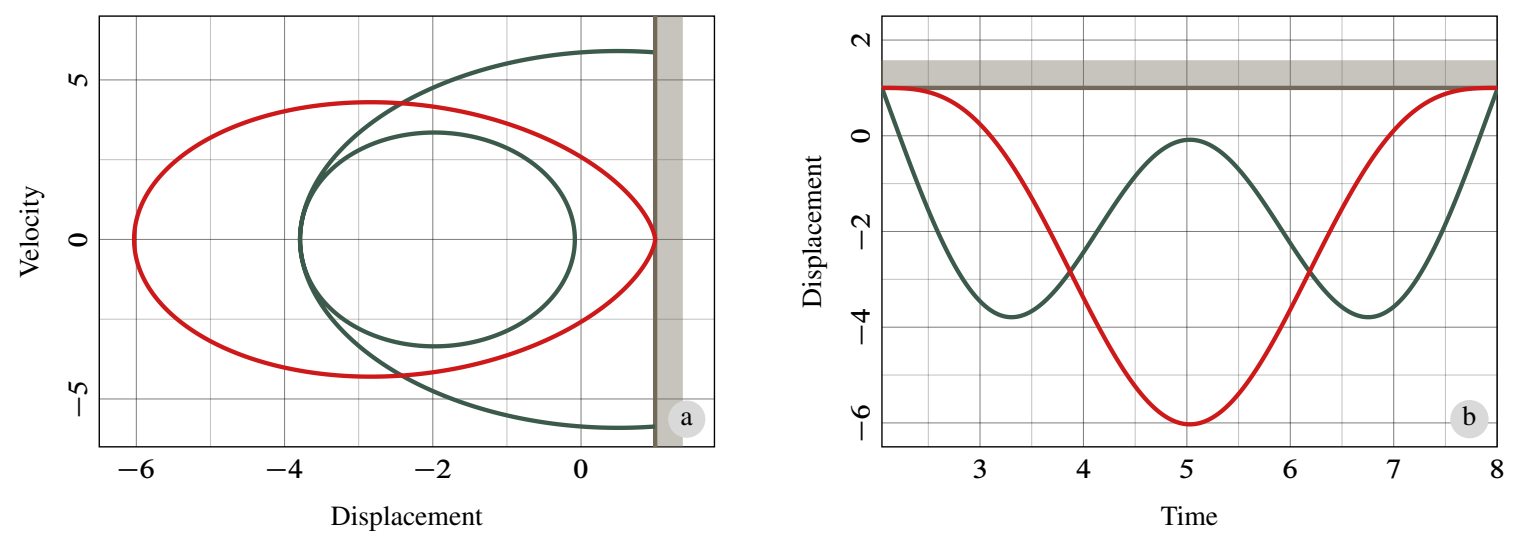

FIGURE 7. (a) Is a 1-IPP or a 1-SPP without sticking phase drawn? (b) Displacements

this particular 1-IPP is now detailed. To this end, generalized 1-SPP and 1-IPP, ie G1-SPP and G1-IPP respectively, are first defined: they are unconstrained 1-SPP and 1-IPP during the free flight and $u_{1}$ as well as $u_{2}$ might exceed $d^{3}$. By definition, a G1-SPP $\mathbf{u}$ satisfies the following requirements:

(1) $s \in Z$,

(2) $T=s+\mathcal{T}$ is the fundamental period with $\mathcal{T}=\mathcal{T}(s)$,

(3) a sticking phase on $] 0 ; \mathcal{T}(s)\left[\right.$ with $u_{1}(0)=d, \dot{u}_{1}(0)=v=v(s), u_{2}(0)=d, \dot{u}_{2}(0)=0$,

(4) a free flight on $] \mathcal{T} ; T\left[\right.$ with $u_{1}(\mathcal{T})=d, \dot{u}_{1}(\mathcal{T})=-v, u_{2}(\mathcal{T})=d, \dot{u}_{2}(\mathcal{T})=0, \mathbf{M u ̈}+\mathbf{K u}=\mathbf{0}$.

The one-to-one correspondence from Figure 2 to Figure 7 is formalized as

$$
\widetilde{\mathbf{u}}(t)=\mathbf{u}(\mathcal{T}+t), \quad 0<t<s,
$$

where $\widetilde{\mathbf{u}}$ is taken $s$-periodic so that $\widetilde{\mathbf{u}}^{-}(0)=\mathbf{u}(0), \widetilde{\mathbf{u}}^{+}(0)=\mathbf{u}(\mathcal{T})$. As a consequence, $\widetilde{\mathbf{u}}$ satisfies

(1) $s$ is the fundamental period,

(2) $\widetilde{u}_{1}^{ \pm}(0)=d, \dot{\widetilde{u}}_{1}^{-}(0)=\dot{u}_{1}(0)=v, \dot{\widetilde{u}}_{1}^{+}(0)=\dot{u}_{1}(\mathcal{T})=-v$,

(3) $\widetilde{u}_{2}^{ \pm}(0)=d, \dot{\widetilde{u}}_{2}^{ \pm}(0)=0$,

(4) a free flight on $] 0 ; s[: \mathbf{M} \ddot{\tilde{u}}+\mathbf{K u}=\mathbf{0}$.

We can check that $\tilde{\mathbf{u}}$ is a G1-IPP. The only surprising condition is $\dot{\widetilde{u}}_{2}(0)=0$ but zero velocity is automatically achieved by a G1-IPP $[4,11]$.

Proposition 5.3 - G1-SPP $\Leftrightarrow \mathbf{G 1}^{-I P P}$. There is a one-to-one correspondence between G1-SPP with a sticking phase for the mass 2 and G1-IPP .

Proof. This is a brief sketch. G1-SPP $\Rightarrow$ G1-IPP was explained previously. Conversely, from a given G1-IPP $P_{\mathrm{p}}$, it is possible to build a sticking phase as in the proof of Theorem 2.1 in Section 4 with a free flight duration $s$ to then define a unique G1-SPP.

The key parameter $s$ appears to be simply the period of the associated G1-IPP . This proposition shows that the set $Z$ corresponds exactly to the set of all G1-IPP . Let us state briefly the correspondence between $Z^{+}$and $Z^{\text {ad }}$ and the corresponding subset of all G1-IPP.

Concerning generalized solutions with a positive velocity at the impact $(v>0)$, it can be said that for

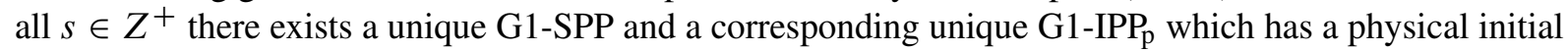
data at the impact time (no violation of the constraint near the impact time). Conversely, if a G1-IPP is such that, at the impact time, the incoming velocity of mass 1 is positive then the period belongs to $Z^{+}$ which corresponds to a unique G1-SPP.

Finally, a 1-SPP, i.e. a G1-SPP satisfying the constraint $u_{2}(t) \leq d$ for all time, is in a unequivocal correspondence with a G1-IPP satisfying the same constraint. This condition is not the constraint to be a 1-IPP since the constraint for 1-IPP is on the mass 1. Figures 2 and 7 show a perfect and rare

\footnotetext{
${ }^{3}$ The G1-IPP in this paper has a counterpart in [11]: it is a G1-IPP where the jump in velocity affects the second mass instead of the first mass here. As such, we know that there is a unique G1-IPP for all positive periods. This is the reason why the formulas in Section 5 are slightly different from [11]. The condition on mass 1 in Theorem 2.1 corresponds to an elastic impact for mass 1 .
} 
correspondence between a 1-SPP and a 1-IPP since the associated G1-IPP satisfies the two constraints $u_{k}(t) \leq d$ for all time and $k=1,2$. As a consequence, 1-SPP are isolated solutions. The reason lies in the fact that the space of G1-IPP is a one-dimensional manifold which intersects $\widetilde{u}_{2}=d$ on a discrete set such that the G1-IPP become isolated. Another possible consequence of Proposition 5.3, which is not further discussed here, is to prove the existence of 1-SPP through the proof of the existence of such particular G1-IPP.

\section{The COUnTABle SET $Z^{ \pm}$}

In order to find admissible solutions, the set $Z^{ \pm}$are defined to encompass the corresponding set of initial data of admissible solution: $\mathcal{V}_{0}^{a d}$. Admissible initial data is defined by a constraint on the associated solution: $u_{2}(t) \leq d$ at least locally near the sticking phase. The problem to satisfy this constraint is only at the end and the beginning of the sticking phase but not for all time. Thus, a question of interest emerges: how large is this set "admissible initial data"? The set of admissible initial data $\mathcal{V}_{0}^{+}$contains $\mathcal{V}_{0}^{\text {ad }}$. In this Section, the sets $Z^{+}$and $Z^{-}$are proven to be countably infinite if some generic assumptions are fulfilled. The proof of Theorem 2.3 is similar for the two sets and only the proof for $Z^{+}$is provided.

6.1. $Z^{ \pm}$is infinite with no resonance. Before stating the main proof with $\omega_{1} / \omega_{2} \notin \mathbb{Q}$, we start with Lemma 6.1 below. First, the orbit $\mathcal{O}$ is defined in the torus $\Pi=\mathbb{R} / 2 \pi \mathbb{Z} \times \mathbb{R} / 2 \pi \mathbb{Z}$ :

$$
\mathcal{O}=\{(x, y)=(\bar{t}, \overline{\rho t}) \mid t>0\}
$$

where $\bar{t}=t+2 \pi \mathbb{Z}$ and $\rho$, a constant.

Lemma 6.1 - Transversality and density. Let $f$ be a $2 \pi$-periodic continuously differentiable function from $\left[0 ; 2 \pi\left[\right.\right.$ to $\left[0 ; 2 \pi\left[\right.\right.$. For any irrational number $\rho$, if $\left(x_{0}, y_{0}=f\left(x_{0}\right)\right)$ located on the curve $\mathcal{C}$ defined by the graph of $f$ satisfies the transversal condition between $\mathcal{C}$ and $\mathcal{O}$, that is

$$
\dot{f}\left(x_{0}\right) \neq \rho
$$

then $\forall \varepsilon>0, \exists t>0$ such that $\overline{\rho t}=f(\bar{t})$ and $\left|\bar{t}-x_{0}\right|<\varepsilon$.

In other words, every point on the curve $\mathcal{C}$ at which the tangent is transverse to the orbit $\mathcal{O}$ is an accumulation point of $\mathcal{O} \cap \mathcal{C}$, see Figure 8. Precisely, the set $\mathcal{O} \cap \mathcal{C}$ is dense in $\{(x, f(x)) \mid \dot{f}(x) \neq \rho\}$.

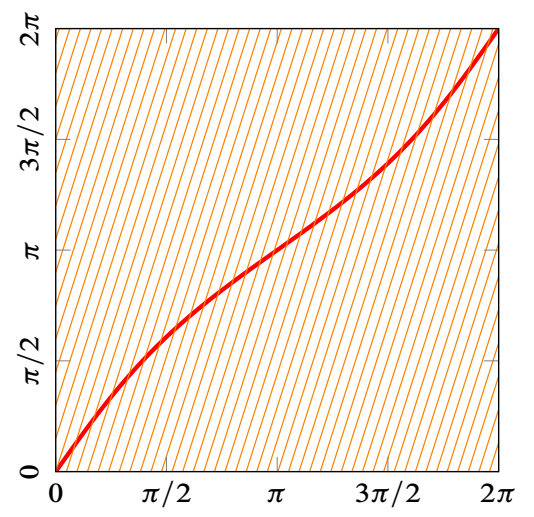

\section{FIgURE 8. Density of $\mathbf{O} \cap \mathbf{H}$ in $\mathbf{H}$}

Moreover, for all $A>0$, the set $\mathcal{O}_{A}=\{(x, y)=(\bar{t}, \overline{\rho t}) \mid t>A\}$ shares the same property.

Proof. Assume $\rho>0$, the cases $\rho=0$ and $\rho<0$ follow immediately.

Since $\dot{f}\left(x_{0}\right) \neq \rho$ and $\dot{f}$ is continuous, there exists $\varepsilon_{0}>0$ small enough such that $\dot{f}(x) \neq \rho$ $\forall x \in\left[x_{0}-\varepsilon_{0} ; x_{0}+\varepsilon_{0}\right]$. Without loss of generality, assume that $\dot{f}(x)>\rho, \forall x \in\left[x_{0}-\varepsilon_{0} ; x_{0}+\varepsilon_{0}\right]$. Since $\mathcal{O}$ is dense in $\Pi, \forall \varepsilon>0$, there exists $t_{0}>0$ such that $z=\left(\overline{t_{0}}, \overline{\rho t_{0}}\right)$ belongs to $\mathcal{O}$ close enough to $\left(x_{0}, y_{0}\right)$, i.e. $\left|\overline{t_{0}}-x_{0}\right|<\varepsilon$ and $\left|\overline{\rho t_{0}}-y_{0}\right|<\varepsilon$ : if $z$ is on the curve $\mathcal{C}$ then $t$ is chosen to be $t_{0}$, else $z$ is above the curve $\mathcal{C}$, i.e. $\overline{\rho t_{0}}>f\left(\overline{t_{0}}\right)$.

We will show that the orbit $\mathcal{O}$ intersects the curve $\mathcal{C}$ inside the box $] x_{0}-\varepsilon_{0} ; x_{0}+\varepsilon_{0}[\times] y_{0}-k \varepsilon_{0} ; y_{0}+$ $k \varepsilon_{0}\left[\right.$ where $k$ is the maximum of $|\dot{f}|$ on $\left[x_{0}-\varepsilon_{0} ; x_{0}+\varepsilon_{0}\right]$ as shown in Figure 9. For this purpose, we 


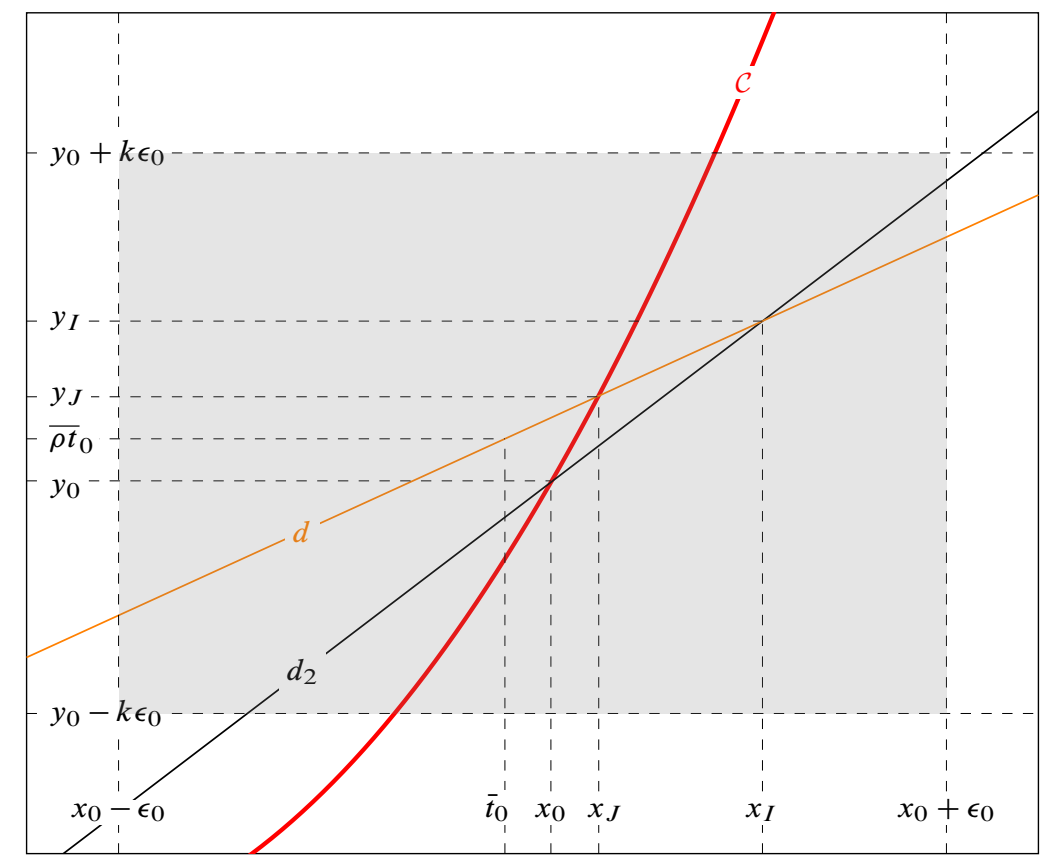

FIGURE 9. Zoom in the box $] x_{0}-\varepsilon_{0} ; x_{0}+\varepsilon_{0}[\times] y_{0}-k \varepsilon_{0} ; y_{0}+k \varepsilon_{0}\left[\right.$ when $\dot{\psi}\left(x_{0}\right)>\rho$

use a line $d_{2}$ under the curve on the right of $\left(x_{0}, y_{0}\right)$. From $p=\min _{\left[x_{0}-\varepsilon_{0} ; x_{0}+\varepsilon_{0}\right]} \dot{f}$, the equation of the line $d_{2}$ with slope $p$ passing through $\left(x_{0}, y_{0}\right)$ is $y=p\left(x-x_{0}\right)+y_{0}$. The line $d$ with slope $\rho$ passing through $\left(\overline{t_{0}}, \overline{\rho t_{0}}\right)$ and associated to the orbit $\mathcal{O}$ is defined by $y=\rho\left(x-\overline{t_{0}}\right)+\overline{\rho t_{0}}$. Let $I\left(x_{I}, y_{I}\right)$ be the intersection of those two lines. Since $p>\rho$, we have

$$
x_{I}=\frac{p x_{0}-y_{0}-\rho \overline{t_{0}}+\overline{\rho t_{0}}}{p-\rho} .
$$

Choosing $\varepsilon$ small enough such that $\varepsilon<\varepsilon_{0}(|p-\rho|) /(\rho+k)$ implies $x_{I} \in\left[x_{0}-\varepsilon_{0} ; x_{0}+\varepsilon_{0}\right]$.

Consider the two curves $d_{2}$ and $\mathcal{C}$ intersecting at $\left(x_{0}, y_{0}\right)$ and satisfying $\dot{f}(x)>p$ for all $x \in$ $\left[x_{0}-\varepsilon_{0} ; x_{0}+\varepsilon_{0}\right]$. Since $p>\rho, d$ intersects $d_{2}$ at $I$. Hence, there exists an intersection of $\mathcal{C}$ and $d$ in the interval $] x_{0} ; x_{I}$ [. In other words, there exists $t>0$ such that $\overline{\rho t}=f(\bar{t})$ and $\left|\bar{t}-x_{0}\right|<\varepsilon_{0}$. The proof for the case $z$ is under the curve $\mathcal{C}$ is similar.

The proof of Theorem 2.3 starts by showing that the set $Z=\{s>0, h(s)=0\}$ is countably infinite. It is true for the set $\left\{\left(\omega_{1} s, \omega_{2} s\right), h(s)=0\right\}$ and will be useful to prove that the set $Z^{+}$of free flight times $s$ with admissible initial velocity $v(s)>0$ is also countably infinite.

Proof. Set $\varphi(t)=\cot (t / 2)$, then $h(s)=\alpha_{1} \varphi\left(\omega_{1} s\right)+\alpha_{2} \varphi\left(\omega_{2} s\right)$ and $w_{1}(s)=\beta_{1} \varphi\left(\omega_{1} s\right)+\beta_{2} \varphi\left(\omega_{2} s\right)$ where $\beta_{j}=b_{1 j}$, and $\alpha_{j}, b_{k j}$ are defined in Equation (2.7). For every $(x, y) \in \Pi=\mathbb{R} / 2 \pi \mathbb{Z} \times \mathbb{R} / 2 \pi \mathbb{Z}$, the two functions $H(x, y)=\alpha_{1} \varphi(x)+\alpha_{2} \varphi(y)$ and $W(x, y)=\beta_{1} \varphi(x)+\beta_{2} \varphi(y)$ correspond to $h(s)=H\left(\omega_{1} s, \omega_{2} s\right), w_{1}(s)=W\left(\omega_{1} s, \omega_{2} s\right)$. In order to simplify, the sets $\mathbf{O}=\left\{\left(\overline{\omega_{1} s}, \overline{\omega_{2} s}\right) \mid s>0\right\}$, $\mathbf{H}=\{(x, y) \in \Pi \mid H(x, y)=0\}$, and $\mathbf{W}=\{(x, y) \in \Pi \mid W(x, y)=0\}$ are defined on the torus $\Pi ; \mathbf{W}^{+}$, $\mathbf{W}^{-}$are denoted as the domains of $\Pi$ where $W(x, y)>0$ and $<0$, respectively.

The set $\mathbf{O}$ is equal to $\mathcal{O}$ with $\rho=\omega_{2} / \omega_{1}$. Consider the map $\gamma: \mathbb{R}^{+} \rightarrow \mathbf{O}, s \mapsto\left(\overline{\omega_{1} s}, \overline{\omega_{2} s}\right)$, then $\gamma$ is bijective for $\omega_{2} / \omega_{1} \notin \mathbb{Q}$ and

$$
\begin{aligned}
\gamma(Z) & =\mathbf{O} \cap \mathbf{H} \\
\gamma\left(Z^{+}\right) & =\mathbf{O} \cap \mathbf{H} \cap \mathbf{W}^{+}
\end{aligned}
$$

Hence, instead of proving the set $Z$ is countably infinite, the stronger result $\overline{\mathbf{O} \cap \mathbf{H}}=\mathbf{H}$ is proven. This implies $\mathbf{O} \cap \mathbf{H}$ is countably infinite. This stronger result shows that $Z^{+}$is countably infinite by pointing out the density of $\mathbf{O} \cap \mathbf{H} \cap \mathbf{W}^{+}$in $\mathbf{H} \cap \mathbf{W}^{+}$and the countable infinity of $\mathbf{H} \cap \mathbf{W}^{+}$.

To show that $\overline{\mathbf{O} \cap \mathbf{H}}=\mathbf{H}$, assume $\alpha_{2} \neq 0$, rewrite $H(x, y)=0$ to have $y=\psi(x)$ where $\psi=$ $\varphi^{-1}(r \varphi)$ and $r=-\alpha_{1} / \alpha_{2}$. 

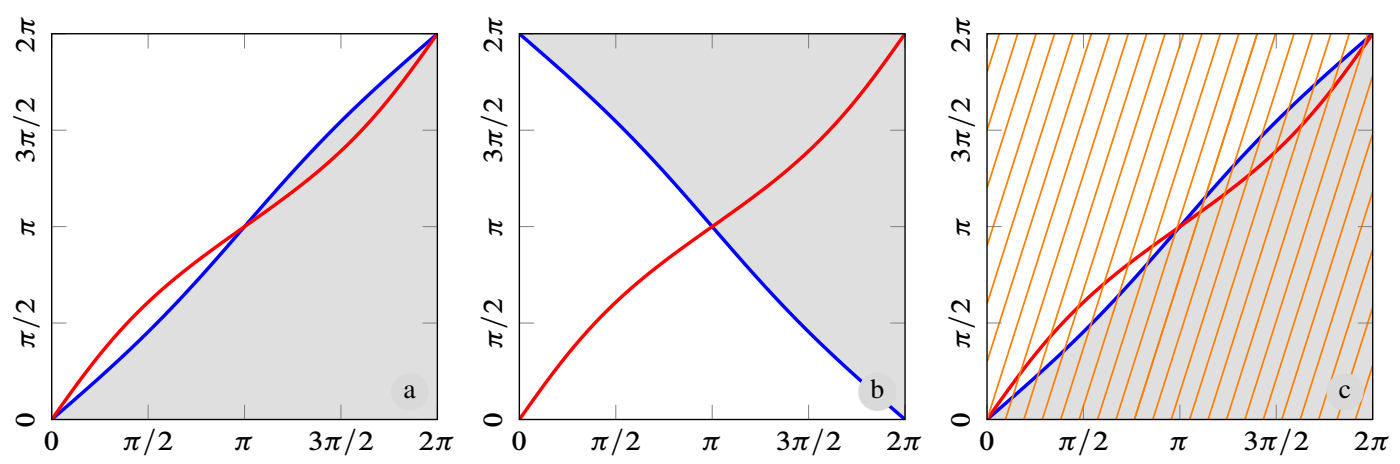

FiguRE 10. (a) or (b) $\mathbf{H} \cap \mathbf{W}^{+}$corresponds to the half of the red curve which lies in the grey domain; (c) The set $\mathbf{O} \cap \mathbf{H} \cap \mathbf{W}^{+}$is the set of all intersections between the red curve and the orange lines within the grey domain

(1) We show that $\dot{\psi} \neq \rho$ almost everywhere. Since $\psi$ is an analytic function on $I=] 0 ; 2 \pi[$, so is $\dot{\psi}$. After simplification, the derivative of $\psi$ becomes

$$
\dot{\psi}=\frac{r\left(1+\varphi^{2}\right)}{1+r^{2} \varphi^{2}}=\frac{1}{r}\left(1+\frac{r^{2}-1}{1+r^{2} \varphi^{2}}\right)
$$

which degenerates to a constant function for $r= \pm 1$. Otherwise, $\dot{\psi}$ is not a constant function and the set $\{x \in I \mid \dot{\psi}(x)=\rho\}$ is empty or countable. Hence, $\dot{\psi} \neq \rho$ holds almost everywhere. It is still true if $\alpha_{2}=0$ since $H(x, y)$ becomes a periodic function of $x$, and $\mathbf{H}$ then degenerates to a vertical line in the torus $\Pi$.

(2) Through Lemma 6.1 where $f=\psi$ is periodic of period $2 \pi$, the set $\mathcal{O}$ is $\mathbf{O}$ where $\rho$ is the ratio $\omega_{2} / \omega_{1}$ and $\mathbf{O} \cap \mathbf{H}$ is dense in $\{(x, y) \in \mathbf{H} \mid \dot{\psi}(x) \neq \rho\}$ follows. In addition, it is proven above that $\dot{\psi} \neq \rho$ almost everywhere, thus $\overline{\mathbf{O} \cap \mathbf{H}}=\mathbf{H}$, since $\mathbf{H}$ is infinite, thus $\mathbf{O} \cap \mathbf{H}$ is countably infinite. In particular, there is a countably infinite set of $s>0$ such that $h(s)=0$.

To complete the proof, we show that $Z^{+}$is countably infinite by proving that $\gamma\left(Z^{+}\right)=\mathbf{O} \cap \mathbf{H} \cap \mathbf{W}^{+}$ is countably infinite. In a similar manner, it is sufficient to show that $\overline{\mathbf{O} \cap \mathbf{H} \cap \mathbf{W}^{+}}=\overline{\mathbf{H} \cap \mathbf{W}^{+}}$and $\mathbf{H} \cap \mathbf{W}^{+}$is an infinite set. If $\beta_{2} \neq 0$, denote $\kappa=-\beta_{1} / \beta_{2}$, the curve $\mathbf{W}$ then corresponds to the function $y=\tilde{\psi}(x)$ where $\tilde{\psi}=\varphi^{-1}(\kappa \varphi)$ which has the same properties with $\psi$. The result still holds if $\beta_{2}=0$ since $\mathbf{W}$ degenerates to the vertical line in $\Pi$. By Assumption 2.1, $r \neq \kappa$ and it follows that $H$ and $W$ cannot coincide and the determinant of the coefficient matrix of the homogeneous system

$$
\begin{aligned}
& H(x, y)=0 \\
& W(x, y)=0
\end{aligned}
$$

is nonzero. Therefore, it has a trivial solution $\varphi(x)=\varphi(y)=0$, i.e. $(\bar{\pi}, \bar{\pi})$ is one intersection between $\mathbf{H}$ and $\mathbf{W}$. Assumption 2.1 is optimal to have $Z^{+}$is infinite. Otherwise, if Assumption 2.1 does not hold, $\mathbf{H}=\mathbf{W}$, thus $\mathbf{H} \cap \mathbf{W}^{+}=\varnothing$ and $Z^{+}$is empty.

Assumption 2.1 implies that $\dot{\psi}(\bar{\pi}) \neq \dot{\tilde{\psi}}(\bar{\pi})$ since $\dot{\psi}(\bar{\pi})=r$ and $\dot{\tilde{\psi}}(\bar{\pi})=\kappa$, thus the curves are transverse. Moreover, since the signs of the derivatives of $\psi$ and $\tilde{\psi}$ depend on the signs of $r$ and $\kappa$, respectively, $\psi$ and $\tilde{\psi}$ are monotonic functions, in which case, $\mathbf{H} \cap \mathbf{W}^{+}$is a half of the curve $\mathbf{H}$ which lies in the region $\mathbf{W}^{+}$(see Figures 10(a) and 10(b)).

As $\mathbf{W}^{+}$is an open set and $\overline{\mathbf{O} \cap \mathbf{H}}=\mathbf{H}$, then $\overline{\mathbf{O} \cap \mathbf{H} \cap \mathbf{W}^{+}}=\overline{\mathbf{H} \cap \mathbf{W}^{+}}$. It follows that $\mathbf{O} \cap \mathbf{H} \cap \mathbf{W}^{+}$ is infinite (Figure 10c.). Hence, $Z^{+}$is infinite which concludes the proof.

6.2. Internal resonances. The situation is much simpler when the ratio $\omega_{1} / \omega_{2}$ is rational. All the functions involved are periodic with the same period and the set $\mathbf{O} \cap \mathbf{H}$ is finite or empty. Thus, the set of initial velocity $\{v(s), s \in Z\}$ is finite which also means that the set of generalized 1-SPP is finite. $Z^{+}$ can be an empty set for instance if $Z=\emptyset$ : with the parameters $\alpha_{1}=1, \alpha_{2}=-1$, and $\omega_{1} / \omega_{2}=2$, the graph of function $h(s)$ is depicted in Figure 11. As a consequence, 1-SPP do not exist in this case. 


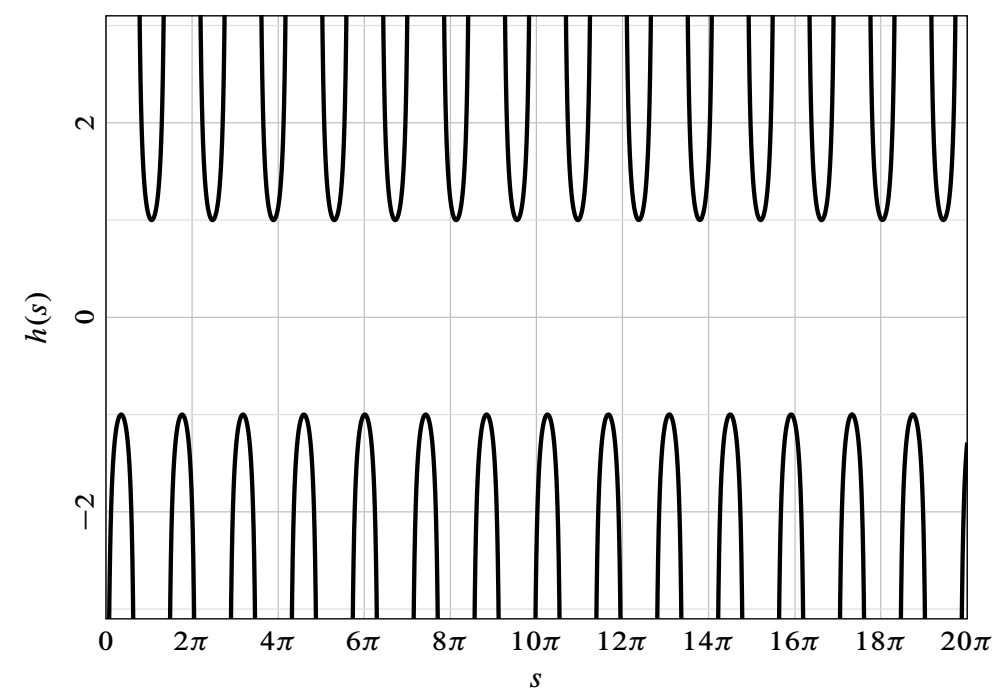

FIGURE 11. Function $h(s)$ when $\omega_{1} / \omega_{2}=2 \in \mathbb{Q}$. To be compared to Figure 3 .

\section{Prestressed structure}

In this Section, the structure of the 1-SPP when $d \leq 0$ is discussed. An argument on the occurrence of the sticking phase is stated in Proposition 7.1. Precisely, sticking phases of unbounded duration can arise besides the solutions with finite sticking phases, when the initial velocity of $m_{1}$ is zero. 1-SPP for $d<0$ and $d=0$ are also explored and illustrated through appropriate numerical examples.

Proposition 7.1. Assume $d \leq 0$. Up to a time translation, periodic solutions with a permanent sticking phase has a one-to-one correspondence to the solutions with initial data:

$$
u_{2}(0)=d, \quad \dot{u}_{2}(0)=0, \quad d \leq u_{1}(0) \leq \bar{u}_{1}:=\frac{k_{2}}{k_{1}+k_{2}} d, \quad \text { and } \quad \dot{u}_{1}(0)=0 .
$$

Otherwise, if

$$
u_{2}(0)=d, \quad \dot{u}_{2}(0)=0, \quad u_{1}(0)=d, \quad \text { and } \quad \dot{u}_{1}(0)>0,
$$

then a sticking phase with finite duration occurs.

The above proposition calls for a few comments:

- for $d=0$, there is only one periodic solution with infinite duration displayed below in Figure 14.

- for $d<0$, the set of periodic solutions with infinite duration is infinite (continuous set).

- It suffices to start at time $t=0$ but not necessarily when $u_{1}$ reaches its minimum. More precisely, a solution has a sticking phase for all $t>0$ if and only if the minimum of $u_{1}$ during the sticking phase is greater or equal to $d$. An easy computation of the 1-dof dynamics of $u_{1}$ during the sticking phase can be written with the energy $\mathbf{E}_{1}$ (see in the proof of Lemma 4.1) as follows.

$$
u_{2}(0)=d, \quad \dot{u}_{2}(0)=0, \quad \text { and } \quad m_{1}\left(\dot{u}_{1}(0)\right)^{2}+\left(k_{1}+k_{2}\right)\left(u_{1}(0)-\bar{u}_{1}\right)^{2} \leq \frac{\left(k_{1} d\right)^{2}}{k_{1}+k_{2}} .
$$

Proof. The proof is a consequence of the proof of Theorem 2.1 together with Remark 4.1 and the 1-dof periodic dynamics of $u_{1}$ around $\bar{u}_{1}$ during the sticking phase. The conditions given in (7.1) (or (7.3)) for $u_{1}$ are just the conditions such that $u_{1}(t) \geq d$ during the period of the "sticking equation" (4.6) then $u_{1}(t) \geq d$ for all $t \geq 0$ and mass 2 remains sticked for all time in the future. For the periodic solution, up to a time translation, it is only assumed that $u_{1}$ reaches its minimum at $t=0$.

In general, the conditions $u_{1}(0)>d$ or $\left\{u_{1}(0)=d\right.$ and $\left.\dot{u}_{1}(0)>0\right\}$ are necessary to enjoy the existence of a sticking phase. However, the latter implies that $u_{1}(t)$ gets strictly smaller than $d$ in the past and by the periodicity in the future, the sticking phase stops. 
7.1. Strictly prestressed structure. The dynamics is explored with $d<0$.

Sticking phase of finite duration. From Proposition 7.1, at the beginning of the sticking phase, the initial data is $[\mathbf{u}(0), \dot{\mathbf{u}}(0)]^{\top}=[d, d, v, 0]^{\top}$ where $v>0$. It directly follows, from Equation (5.15) when $d<0$, that the admissible initial data is found in the set $Z^{-}$instead of $Z^{+} . Z^{-}$is also countably infinite as stated in Theorem 2.3. In a manner similar to the case $d>0$, an infinite set of admissible initial data is expected when $d<0$.

A 1-SPP is depicted in Figure 12 where $d=-1$; the positive initial velocity is $v \approx 2.26$. With a period $T \approx 5.42$, the sticking phase occurs until $\mathcal{T} \approx 1.58$ and is then followed by a free flight of duration $s \approx 3.84$.
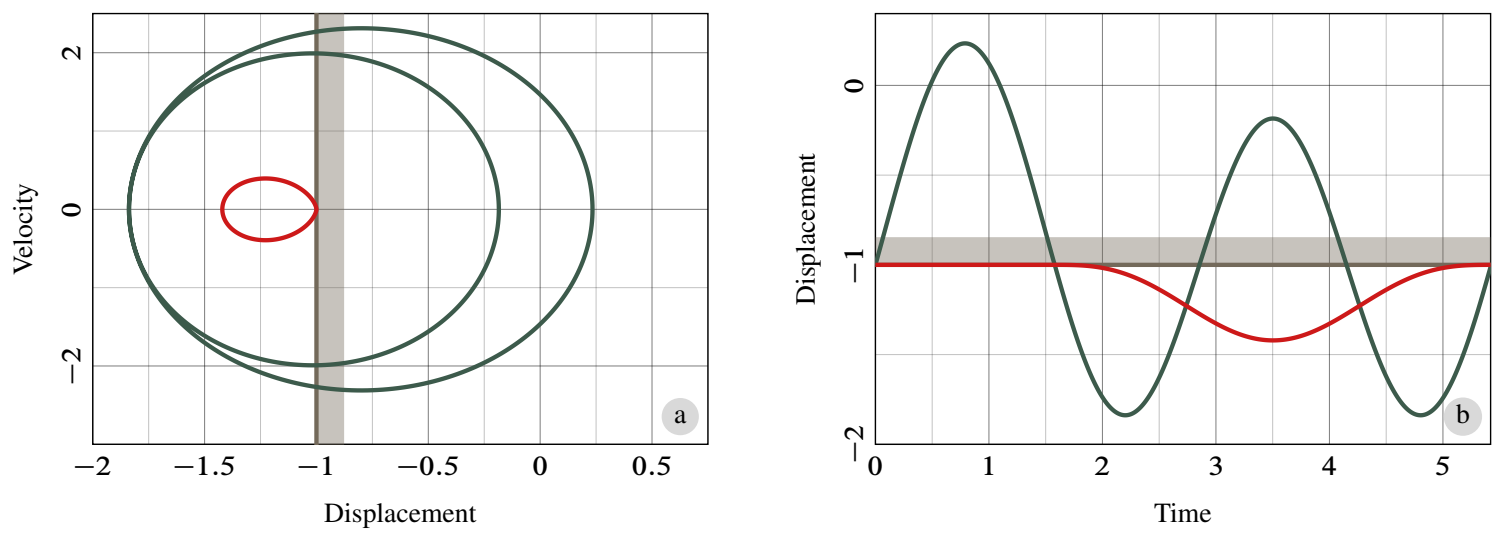

FIGURE 12. 1-SPP with finite sticking phase for $d=-1$ and $v>0, m_{1}=1.0 ; m_{2}=$ $6.0 ; k_{1}=1.0 ; k_{2}=4.0$ and initial data $U(0)=[-1,-1,2.2686626,0]$ : (a) Orbits.

(b) Displacements

Sticking phase of infinite duration. The initial data corresponding to the periodic solution with the largest $u_{1}$ in magnitude is $[\mathbf{u}(0), \dot{\mathbf{u}}(0)]^{\top}=[d, d, 0,0]^{\top}$. The first mass then follows the oscillation around its new equilibrium $\bar{u}_{1}$. Moreover, 0 is the minimum point of $u_{1}$, thus $u_{1}(t) \geq d$ for all $t$. By Theorem 2.1, it follows that the sticking phase never ends. This argument is illustrated in Figure 13.
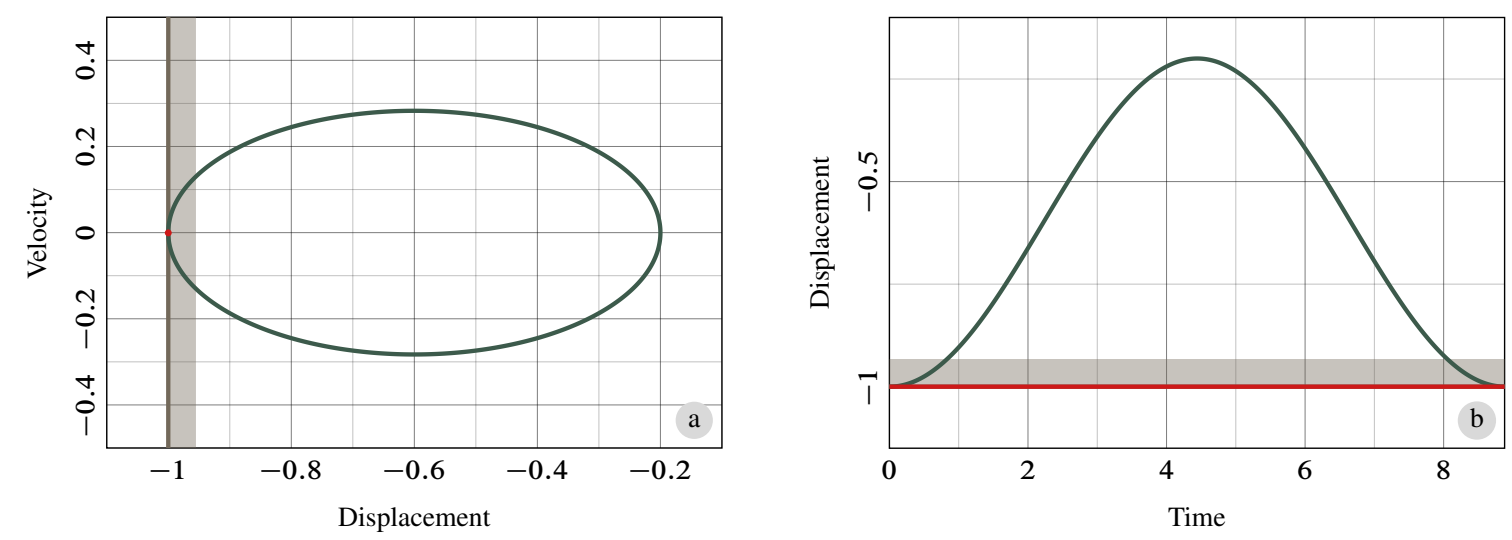

FIGURE 13. Sticking phase of infinite duration for $d=-1<0$ and $v=0, m_{1}=10$; $m_{2}=6 ; k_{1}=2 ; k_{2}=3$ with initial data $U(0)=[-1,-1,0,0]$. (a) Orbits. (b) Displacements 
7.2. Statically grazing system. The dynamics is explored with $d=0$.

Finite duration sticking phase. From Proposition 7.1, the sticking phase of finite duration exists if the initial data satisfies $u_{2}(0)=0, \dot{u}_{2}(0)=0, u_{1}(0)=0$, and $\dot{u}_{1}(0)=v>0$. The set of free flight time $s$ is found from Equation (5.14) where $d=0$, i.e. $v w_{1}(s)=0$ and $v w_{2}(s)=0$. Hence, $v$ is arbitrarily positive and $s$ satisfies $h(s)=w_{1}(s)-w_{2}(s)=0$ and $w_{2}(s)=0$ or

$$
\left[\begin{array}{ll}
\alpha_{1} & \alpha_{2} \\
\beta_{1} & \beta_{2}
\end{array}\right]\left[\begin{array}{l}
\varphi\left(\omega_{1} s / 2\right) \\
\varphi\left(\omega_{2} s / 2\right)
\end{array}\right]=\left[\begin{array}{l}
0 \\
0
\end{array}\right]
$$

Through Assumption 2.1, this linear system has the unique solution

$$
\begin{aligned}
& \varphi\left(\omega_{1} s / 2\right)=0 \\
& \varphi\left(\omega_{2} s / 2\right)=0
\end{aligned}
$$

where $\varphi(t)=\cot (t / 2)$. It follows that

$$
\frac{\omega_{1}}{\omega_{2}}=\frac{2 k+1}{2 l+1} \quad \text { with } \quad k, l \in \mathbb{Z}
$$

condition which loosely speaking represents half of the rationals. It should be satisfied to observe a sticking phase of finite duration when $d=0$ while the initial velocity of mass $m_{1}$ can be chosen arbitrarily positive. Such a 1-SPP when $\omega_{1} / \omega_{2}=1 / 5$ is shown in Figure 14 .
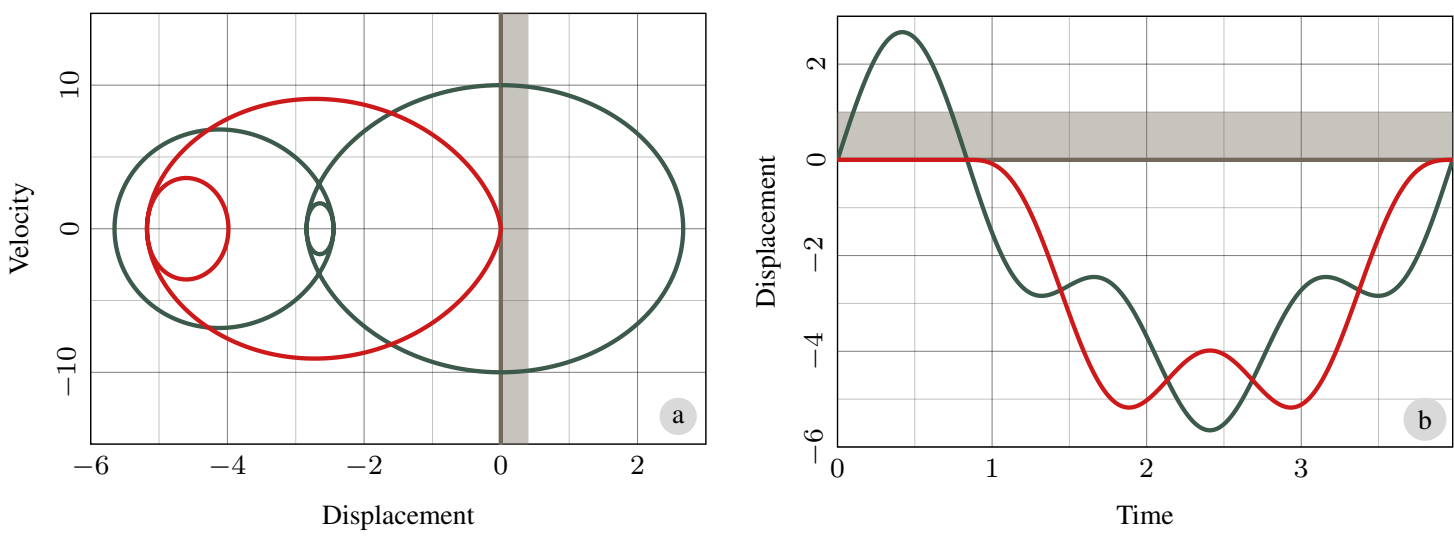

FIGURE 14. 1-SPP with finite sticking phase for $d=0$ and $v=10$ (any arbitrary positive number is acceptable). (a) Orbits. (b) Displacements.

Infinite duration sticking phase. The unique initial data in this case is $[\mathbf{u}(0), \dot{\mathbf{u}}(0)]^{\top}=[0,0,0,0]^{\top}$. The equilibrium $\mathbf{u} \equiv \mathbf{0}$ is a solution in which mass $m_{2}$ always grazes with the wall since the two masses stay at their equilibrium position when $d=0$ and $\dot{u}_{1}(0)=0$.

It should thus be noted that generically, there is no 1-SPP except the equilibrium when $d=0$.

\section{CONCLUSION}

The free dynamics of a two-degree-of-freedom linear oscillator subject to a unilateral constraint on one of its mass is investigated. Generically, a Newton-like impact law has to be incorporated in the formulation to guarantee well-posedness. In this work, periodic orbits with one sticking phase per period (1-SPP) are considered: it is shown that they are independent of the impact law. They might not always exist and whenever they exist, they are isolated as opposed to one-impact-per-period solutions (1-IPP) known to be organized on manifolds [11]. Also, they cannot be obtained through usual perturbation methods.

The whole set of 1-SPP is characterized by only one parameter belonging to a discrete set: the free flight duration of the trajectories. This parameter belongs to a countable set which can be empty, or even infinite in some circumstances.

A systematic numerical procedure is designed to find all possible 1-SPP. It involves two numerical steps:

(1) finding the roots of an explicit quasi-periodic function, and 
(2) checking that the corresponding closed-form trajectory satisfies the unilateral condition on the whole period of motion.

Many examples are presented but the existence of 1-SPP remains an open problem. The situation is worse: conditions for the non-existence of 1-SPP are provided. However, under generic assumptions on the mass and stiffness matrices, a countable infinite set of initial data including all the initial data of 1-SPP can be exhibited. The closed-forms emanating from this set (of initial data) satisfy the unilateral constraint at least near the sticking phase. The prestressed structure is also explored. The picture is similar except that 1-SPP with infinite sticking time are also found.

Extension to $n$ degrees-of-freedom with $n>2$ is not straightforward: the symmetry $\mathbf{u}(t)=\mathbf{u}(-t)$, property heavily used in this work, is unknown and the sticking dynamics is more complicate, the sticking system has dimension $n-1>1$.

\section{REFERENCES}

[1] Vincent Acary and Bernard Brogliato. Numerical Methods for Nonsmooth Dynamical Systems, volume 35 of Lecture Notes in Applied and Computational Mechanics. Springer, 2008. [inria-00423530].

[2] Robert Adams. Sobolev Spaces, volume 65 of Pure and Applied Mathematics. Academic Press, 1975.

[3] Ricardo Alzate, Mario di Bernardo, Umberto Montanaro, and Stefania Santini. Experimental and numerical verification of bifurcations and chaos in cam-follower impacting systems. Nonlinear Dynamics, 50(3):409-429, 2007. [hal-01304369].

[4] Patrick Ballard. The dynamics of discrete mechanical systems with perfect unilateral constraints. Archive for Rational Mechanics and Analysis, 154(3):199-274, 2000. [hal-00111308].

[5] Jérôme Bastien, Frédéric Bernardin, and Claude-Henri Lamarque. Systèmes dynamiques discrets non réguliers déterministes ou stochastiques : applications aux modèles avec frottement ou impact. Hermès Science, Paris, 2012.

[6] Chris Budd and Felix Dux. Chattering and related behaviour in impact oscillators. Philosophical Transactions of the Royal Society London A, 347(1683):365-389, 1994. [hal-01304424].

[7] Mario di Bernardo, Chris Budd, Alan Champneys, and Piotr Kowalczyk. Piecewise-smooth dynamical systems: theory and applications. Applied Mathematical Sciences. Springer Science \& Business Media, London, 2008.

[8] David Doyen, Alexandre Ern, and Serge Piperno. Time-integration schemes for the finite element dynamic Signorini problem. SIAM Journal of Scientific Computing, 33(1):223-249, 2011. [hal-00440128].

[9] Sokly Heng. Nonlinear normal modes of impact oscillators. Internship report, 2013.

[10] Stéphane Junca, Huong Le Thi, Mathias Legrand, and Anders Thorin. Impact dynamics near unilaterally constrained grazing orbits. In 9th European Nonlinear Dynamics Conference (ENOC), Budapest, Hungary, 2017. [hal-01562154].

[11] Mathias Legrand, Stéphane Junca, and Sokly Heng. Nonsmooth modal analysis of a $n$-degree-of-freedom system with a purely elastic impact law. Communications in Nonlinear Science and Numerical Simulation, 45:190-219, April 2017. [hal-01185980v2].

[12] Arne Nordmark and Petri Piiroinen. Simulation and stability analysis of impacting systems with complete chattering. Nonlinear Dynamics, 58(1-2):85-106, 2009. [hal-01304375].

[13] Madeleine Pascal. Dynamics and stability of a two-degree-of-freedom oscillator with an elastic stop. Journal of Computational and Nonlinear Dynamics, 1(1):94-102, 2005. [hal-00342874].

[14] Danilo Percivale. Uniqueness in the elastic bounce problem. Journal of Differential Equations, 56(2):206-215, 1985. [hal-01294061].

[15] Valery Pilipchuk. Impact modes in discrete vibrating systems with rigid barriers. International Journal of Non-Linear Mechanics, 36(6):999-1012, 2001. [hal-01295245].

[16] Michelle Schatzman. A class of nonlinear differential equations of second order in time. Nonlinear Analysis: Theory, Methods \& Applications, 2(3):355-373, 1978. [hal-01294058].

[17] Anders Thorin, Pierre Delezoide, and Mathias Legrand. Nonsmooth modal analysis of piecewise-linear impact oscillators. SIAM Journal on Applied Dynamical Systems, 16(3):1710-1747, 2017. [hal-01298983].

[18] Anders Thorin, Pierre Delezoide, and Mathias Legrand. Periodic solutions of $n$-dofs autonomous vibro-impact oscillators with one lasting contact phase. Nonlinear Dynamics, 90(3):1771-1783, 2017. [hal-01505888].

[19] Anders Thorin, Mathias Legrand, and Stéphane Junca. Nonsmooth modal analysis: investigation of a 2-dof spring-mass system subject to an elastic impact law. In ASME International Design Engineering Technical Conferences and Computers and Information in Engineering Conference: 11th International Conference on Multibody Systems, Nonlinear Dynamics, and Control, Boston, Massachusetts, 2015. [hal-01185973]. 